University of Nebraska - Lincoln

DigitalCommons@University of Nebraska - Lincoln

\title{
Local and segmental motions of the mobile amorphous fraction in semi-crystalline polylactide crystallized under quiescent and flow-induced conditions
}

\author{
Xavier Monnier \\ Normandie Université, xavier.monnier1@univ-rouen.fr \\ Laurence Chevalier \\ Normandie Université \\ Antonello Esposito \\ Normandie Université \\ Lucia Fernandez-Ballester \\ University of Nebraska-Lincoln, lucia.fernandez@unl.edu \\ Allisson Saiter \\ Normandie Université \\ Follow this and additional works at: https://digitalcommons.unl.edu/mechengfacpub \\ See next page for additional authors \\ Part of the Mechanics of Materials Commons, Nanoscience and Nanotechnology Commons, Other \\ Engineering Science and Materials Commons, and the Other Mechanical Engineering Commons
}

Monnier, Xavier; Chevalier, Laurence; Esposito, Antonello; Fernandez-Ballester, Lucia; Saiter, Allisson; and Dargent, Eric, "Local and segmental motions of the mobile amorphous fraction in semi-crystalline polylactide crystallized under quiescent and flow-induced conditions" (2017). Mechanical \& Materials Engineering Faculty Publications. 342.

https://digitalcommons.unl.edu/mechengfacpub/342

This Article is brought to you for free and open access by the Mechanical \& Materials Engineering, Department of at DigitalCommons@University of Nebraska - Lincoln. It has been accepted for inclusion in Mechanical \& Materials Engineering Faculty Publications by an authorized administrator of DigitalCommons@University of Nebraska Lincoln. 


\section{Authors}

Xavier Monnier, Laurence Chevalier, Antonello Esposito, Lucia Fernandez-Ballester, Allisson Saiter, and Eric Dargent 


\title{
Local and segmental motions of the mobile amorphous fraction in semi-crystalline polylactide crystallized under quiescent and flow-induced conditions
}

\author{
Xavier Monnier, ${ }^{1}$ Laurence Chevalier, ${ }^{1}$ \\ Antonella Esposito, ${ }^{1}$ Lucia Fernandez-Ballester, ${ }^{2}$ \\ Allisson Saiter, ${ }^{1}$ and Eric Dargent ${ }^{1}$ \\ 1 Normandie Univ, UNIROUEN Normandie, INSA Rouen, CNRS, \\ Groupe de Physique des Matériaux, 76000 Rouen, France \\ 2 Department of Mechanical and Materials Engineering, \\ University of Nebraska at Lincoln, Lincoln, NE 68588, \\ United States \\ Corresponding author -X. Monnier, email xavier.monnier1@univ-rouen.fr
}

\begin{abstract}
The molecular dynamics of the constrained and unconstrained mobile amorphous fractions in semicrystalline polylactide (PLA) was investigated in the presence of both flow-induced crystalline structures and spherulites by fast scanning calorimetry (FSC) through cooperativity and physical aging concepts. First, the shear rate conditions leading to flow-induced crystallization were characterized by examining the relaxation of shear-induced precursors. At a temperature of $150^{\circ} \mathrm{C}$, the critical relaxation time is so long that cooling down the sheared melt to the crystallization temperature does not affect significantly the precursors. Therefore, highly oriented structures develop. The arrangement of the crystalline fraction, either in flow-induced crystalline structures or spherulites, has no influence on the molecular dynamics of the mobile amorphous fraction, whereas the crystallinity degree was proved to play a significant role. Two kinds of molecular dynamics were
\end{abstract}


distinguished, respectively associated to the $\alpha$ and secondary relaxations. The molecular dynamics related to the $\alpha$ relaxation of the mobile amorphous fraction was investigated in terms of cooperativity: a clear dependence on the coupling and the confinement by the crystals was observed, because a classic decrease of the cooperativity length was found when comparing amorphous to semi-crystalline PLA. On the contrary, the molecular dynamics related to the secondary relaxations of the mobile amorphous fraction was not modified by the presence of the crystals, as observed from close physical aging investigations below the glass transition. The different length-scale of the $\alpha$ and secondary relaxations seems to be the reason why the crystalline environment influences the macromolecular motions rather than the molecular or local motions.

Keywords: Polylactide, Amorphous fractions, Flow-induced crystallization, Spherulite, Fast scanning calorimetry, Physical aging

\section{Introduction}

When polymers crystallize, the macromolecular chains that initially are in 3D random coil conformations arrange segments of their chains into lamellar crystals. ${ }^{[1]}$ Semi-crystalline polymers can be obtained by either quiescent or prestressed crystallization pathways. Crystallization is quiescent when the process is only thermally activated, whether performed upon cooling from the melt or upon heating from the glassy state to a given temperature. ${ }^{[2,3]}$ Quiescent crystallization is also possible in the presence of nucleating agents, which allow tailoring crystal morphology and lamellae orientation. ${ }^{[4]}$ Prestressed crystallization emerges from specific conditions, such as memory effects, ${ }^{[5]}$ cross-nucleation ${ }^{[6,7]}$ or thermo-mechanical history. ${ }^{\left[{ }^{[8]}\right.}$ The latter case is of importance for processing, as semi-crystalline polymers are frequently subjected to intense shear and/or elongational flows in the molten state prior to crystallization. Over the last few decades, numerous works have investigated the initial stages of flow-induced crystallization ${ }^{[9-14]}$ as well as the final oriented semicrystalline microstructure. [15-19] Melt flow of sufficient intensity results in local alignment and stretch of chain segments which can induce the formation of oriented nucleation precursors. Flow-induced precursors are thought to be metastable quasicrystalline aggregates that can persist at high temperatures for long times, even above the nominal melting point. The main consequences of the presence of oriented flow-induced precursors are a significant acceleration of the crystallization kinetics and a transition from an overall isotropic morphology (spherulites) to anisotropic flow-induced crystalline structures. As a result of the oriented crystalline morphology that can develop after flow, final physical properties are also significantly impacted. 
Flow-induced crystallization has been frequently investigated on polyolefins-particularly polyethylene and polypropylene-because of their commercial relevance. Due to environmental concerns, several studies have recently focused on flow-induced crystalline structures in polylactide (PLA), ${ }^{[20-25]}$ a biosourced and biodegradable thermoplastic polyester. Quiescent crystallization of PLA leads to the concomitant formation of highly ordered $\alpha$ crystals and less ordered $\delta$ crystals. ${ }^{[26]}$ It is established that quiescent crystallization performed at temperatures higher than $120^{\circ} \mathrm{C}$ leads to $\alpha$ crystals, whereas below $100{ }^{\circ} \mathrm{C} \delta$ crystals are obtained. In the intermediate range of temperature, concomitant crystallization of both $\alpha$ and $\delta$ crystals occurs. ${ }^{[27]}$ The influence of shear or elongational flow onto the crystalline structures that develop in PLA has been mainly investigated in order to improve physical attributes, such as oxygen barrier ${ }^{[28]}$ or mechanical properties. ${ }^{[29]}$ It should be mentioned that, in some cases, semicrystalline microstructures similar to flow-induced crystalline structures have also been obtained in PLA-based composite materials ${ }^{[30,31]}$ and in PLA-based formulations containing nucleating agents ${ }^{[32,33]}$ or nano-fibrils. ${ }^{[34]}$

Most of the existing studies, whether on polyolefins or PLA, are focused on characterizing the crystalline fraction, and not on the confinement of the amorphous fractions by flow-induced crystalline structures. In addition, the lifetime of flow-induced precursors in PLA has not yet been explored. The recent development of fast scanning calorimetry (FSC) has represented a real breakthrough for the study of polymer crystallization, ${ }^{[35,36]}$ polymer melting ${ }^{[37,38]}$ and amorphous polymer properties. ${ }^{[39,40]}$ Fast scanning techniques allow analyzing a few ng of a sample by using rates as fast as thousands of Kelvin per second. ${ }^{[41]}$ As a consequence, the analysis of amorphous fractions confined by crystals at the scale of semi-crystalline superstructures is now possible.

The crystallization of polymers is a kinetic process that results in the formation of nano-phasic structures where crystalline and amorphous regions coexist. ${ }^{[42]}$ The final morphology results from a competition between crystallization and vitrification. For decades now, most semi-crystalline polymers have been described in the literature with a three-phase model. ${ }^{[43,44]}$ In fact, between the crystalline and the amorphous fractions, an incomplete decoupling may exist that defines an additional fraction called the rigid amorphous fraction (RAF) ${ }^{[45,46]}$. This fraction is due to the fact that polymer chains are much longer than the typical dimensions of polymer crystals. ${ }^{[47,48]}$ RAF is commonly defined by opposition to the mobile amorphous fraction (MAF) in that its mobility is drastically restricted due to the geometrical constraints at the interface with the crystals. ${ }^{[49,50]}$ Regarding the $M A F$, constrained and unconstrained MAF-depicted as intra- and interspherulitic amorphous phases respectively-have recently been reported 
for semicrystalline polyesters such as PET ${ }^{[51]}$ and PLA. ${ }^{[43,52,53]}$ Constrained and unconstrained MAF were revealed by differential scanning calorimetric measurements through the concept of cooperative rearranging regions $(\mathrm{CRR})^{[54]}$ as well as structural relaxation (i.e. physical aging). ${ }^{[55]}$

The purpose of this paper is to investigate the relaxation of shear-induced precursors leading to flow-induced crystallization of PLA, so that the amorphous fractions constrained within different semi-crystalline microstructures can be investigated through glass transition and physical aging by means of FSC. The presence of crystals affects the molecular dynamics of the MAF; therefore, a question arises: what is the role of crystalline morphology?

\section{Materials and methods}

\subsection{Materials and sample preparation}

PLA pellets (grade $4042 D$, containing about $96 \%$ of L-lactide and $4 \%$ of Dlactide) were provided by NatureWorks. The number-average and weightaverage molecular weights were $\left\langle\mathrm{M}_{\mathrm{n}}\right\rangle=116 \mathrm{kDa}$ and $\left\langle\mathrm{M}_{\mathrm{w}}\right\rangle=188 \mathrm{kDa}$ respectively, as measured by gel permeation chromatography (GPC). Prior to use, PLA pellets were dried overnight at $60{ }^{\circ} \mathrm{C}$ to prevent degradation. The pellets were then thermo-compressed between two hot plates during $5 \mathrm{~min}$ at a pressure of $1 \mathrm{bar}$ and a temperature of $180^{\circ} \mathrm{C}$. The temperature was selected below the equilibrium melting temperature $T_{m}{ }^{0}$ (ranging from $199^{\circ} \mathrm{C}$ to $207^{\circ} \mathrm{C}{ }^{[56]}$ ) and above the nominal melting temperature $T_{\mathrm{m}}$ (ranging from 160 to $170{ }^{\circ} \mathrm{C}[57,58]$ ). The samples were then quickly quenched in cold water and dried, obtaining self-standing films with thicknesses ranging between 200 and $300 \mu \mathrm{m}$. A single glass fiber with a diameter of $17 \pm$ $1 \mu \mathrm{m}$ was sandwiched between two PLA films and the assembly was then placed between two microscope cover-glasses.

\subsection{Procedure for fiber pulling experiments}

A homemade device was used to pull the fiber through the molten polymer at a constant linear velocity of $10 \mathrm{~mm} \mathrm{~s}^{-1}$ for $1 \mathrm{~s}$. The shear rate at the interface between the fiber and the melt was estimated to be close to $1000 \mathrm{~s}^{-1}$, according to Monasse et al. ${ }^{[15]}$ The different steps of the fiber pulling procedure were performed as follows. First, the as-prepared sandwich was transferred into a Mettler FP 82 hot stage and held at $180{ }^{\circ} \mathrm{C}$ for 5 min to erase any previous thermal and mechanical history. Then, the assembly was cooled to a given pull-out temperature $T_{\text {po }}$ and held there for 3 min before pulling the fiber to impose flow onto the melt. From this point, 
two different procedures were followed: one to investigate the precursors' relaxation (protocol 1 ) and another one to probe the amorphous fraction present in flow-induced highly oriented structures (protocol 2). For experiments that followed protocol 1 , shear rate was applied by pulling the fiber at different temperatures $\left(\mathrm{T}_{\mathrm{po}}=157.5^{\circ} \mathrm{C}, 160^{\circ} \mathrm{C}, 162.5^{\circ} \mathrm{C}, 165^{\circ} \mathrm{C}, 167.5\right.$ ${ }^{\circ} \mathrm{C}$ and $170^{\circ} \mathrm{C}$ ). The assembly was then held at $\mathrm{T}_{\text {po }}$ for a specific relaxation time-ranging from few seconds to few hours-prior to quenching to a suitable crystallization temperature $\left(T_{c}=130^{\circ} \mathrm{C}\right)$. Depending on the extent of precursors' relaxation, either a spherulitic or an oriented flow-induced crystalline structures was observed as crystallization proceeded. For experiments that followed protocol 2, shear precursors were induced by fiber pulling at $\mathrm{T}_{\text {po }}=150^{\circ} \mathrm{C}$. Next, the sample was immediately quenched to the same crystallization temperature of $\mathrm{T}_{\mathrm{c}}=130^{\circ} \mathrm{C}$ to prevent any significant precursor relaxation. Then, isothermal crystallization was allowed to proceed for $90 \mathrm{~min}$. Note that for protocol 2, the fiber was completely pulled out from the melt in order to allow the sample preparation that is required for subsequent thermal analysis. For more clarity, schematization of both protocols are displayed in supporting information.

\subsection{Morphological observations}

Morphological observations during and after isothermal crystallization were performed with a universal EPI-illuminator polarized optical microscope (POM) equipped with Nikon M Plan $\times 2.5 / \times 5 / \times 10$ objective lenses and connected with a digital camera system from Nikon Corporation in transmission mode.

Additional observations of the flow-induced crystalline structures were performed by scanning electronic microscopy (SEM). Flow-induced crystalline structures were isolated by ultramicrotomy on an Ultracut UCT Leicamicrosystem with a glass knife; this step allowed reaching the center of the structure by cutting along the pulling direction. The crystalline structure was then isolated by etching the sample in a water-methanol (1:2 by volume) solution containing $0.025 \mathrm{~mol} / \mathrm{L}$ of sodium hydroxide at room temperature during $24 \mathrm{~h}$, followed by rinsing in distilled water. Finally, the sample was coated with $10 \mathrm{~nm}$ of gold with a Cressington Sputter Coater prior to observations with a Dual-BEAM LEO1530-ZEISS scan electron microscope at an accelerating voltage of $5 \mathrm{kV}$.

\subsection{Fast scanning calorimetric measurements}

FSC analyses were performed using a power compensation twin-type chipbased fast scanning calorimeter Flash DSC 1 (Mettler Toledo) equipped with a Huber TC100 intra-cooler. Cross sections of both flow-induced crystalline 
structures and spherulites were obtained by ultramicrotomy. Serial sections of $500 \mathrm{~nm}$ thickness were obtained with an Ultracut UCT Leica-microsystem with a dry-glass knife. The samples were placed on the sensitive area of a MultiSTAR UFS 1 MEMS chip sensor. A thin layer of silicone oil was used to promote adhesion of the sample onto the chip and to improve thermal contact. Samples were continuously flushed with a $20 \mathrm{~mL} \mathrm{~min}^{-1}$ flow of gaseous nitrogen to prevent condensation from environmental moisture and to ensure temperature control. Sample mass was estimated from the step change in heat flow at the glass transition: the heat capacity step $\Delta C_{p}$ obtained by FSC at $\mid \beta_{c} I=\beta_{h}=1500 \mathrm{~K} \mathrm{~s}^{-1}$ was compared to the value obtained by conventional DSC at $\left|\beta_{c}\right|=\beta_{h}=0.17 \mathrm{~K} \mathrm{~s}^{-1}$, that is to say $0.51 \mathrm{~J} /(\mathrm{g} . \mathrm{K})$ for a completely amorphous PLA according to the literature. ${ }^{58]}$

Before FSC experiments, the shape of the samples was observed by optical microscopy on a microscope cover-glass in transmission mode. After FSC experiments, the shape of the samples adhered on the chip sensor was examined in reflection mode. The perimeter and the surface area were measured with the software ImageJ.

\section{Results and discussion}

\subsection{Quiescent and prestressed crystallization of PLA}

Flow-induced crystallization in PLA has been reported in the presence of steady ${ }^{[20,24]}$ or oscillating ${ }^{[22,25]}$ shear conditions during injection molding, and by pulling fibers within a polymer melt. ${ }^{[21,31]}$ The latter procedure is the easiest way to produce shear flows with shear rates up to $\approx 1500 \mathrm{~s}^{-1}$. [31] However, to our knowledge, the lifetime of shear-induced precursors in PLA has not been previously investigated. Due to their metastability, the existence of shear-induced precursors is time and temperature dependent, as already observed for other polymers such as PP, PBu, PEO or PS. ${ }^{[11,12] ~ I n ~}$ this work, the lifetime and relaxation behavior of PLA shear-induced precursors was investigated in the temperature range where melting of semicrystalline PLA is typically reported ${ }^{[57,58]}$ to mimic flow conditions typical of industrial processing. The relaxation temperature map in Fig. 1 depicts the relaxation time of PLA shear-induced precursors $\left(\mathrm{t}^{\star}\right)$ measured at different pull-out temperatures $\left(T_{p o}\right)$ and the type of morphology that develops after cooling to the crystallization temperature (protocol 1). Longer relaxation times, i.e. above the "transition zone" in Fig. 1, lead to only spherulites because the precursors completely relax, whereas for shorter relaxation times the precursors are still present, which results in cylindrical flow-induced crystalline structures near the fiber, i.e. in the region that was subjected to the highest shear rate. At intermediate times, i.e. in the "transition zone", 


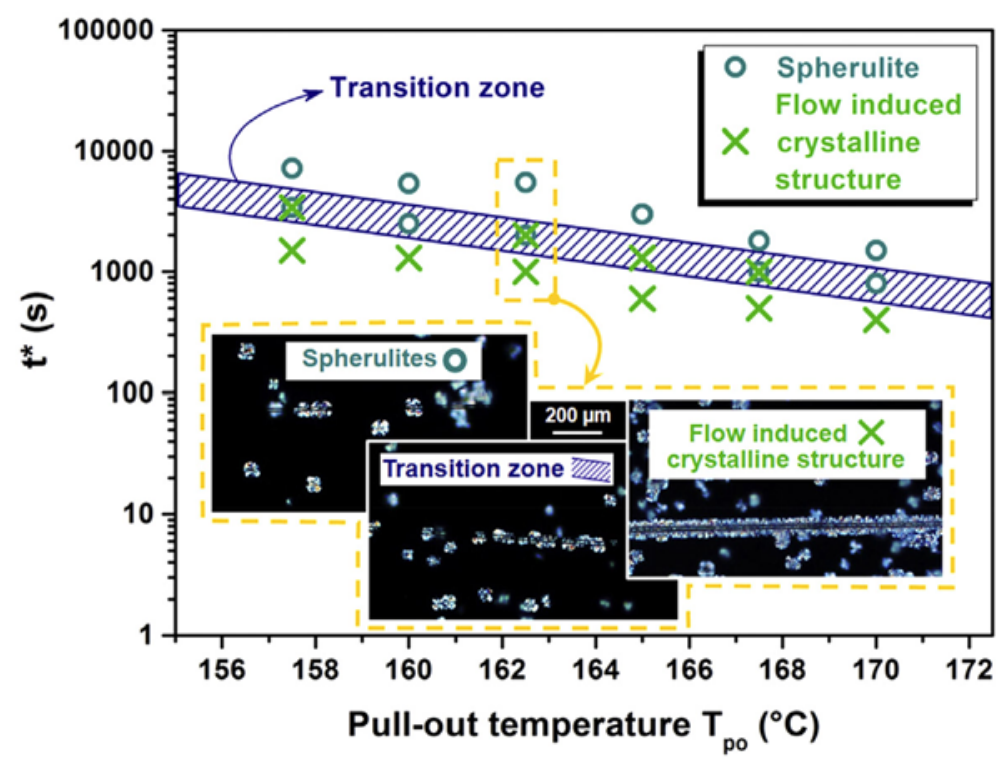

Fig. 1. Holding time $\left(\mathrm{t}^{\star}\right)$, after pulling the fiber, as a function of the pull-out temperature (Tpo). The holding time $t^{*}$ is related to the relaxation of the precursors, and their lifetime is then observed after crystallization at $\mathrm{T}_{\mathrm{c}}=130^{\circ} \mathrm{C}$. For "Ionger" relaxation times (circles), the precursors relax and spherulites (SPE) are obtained. For "shorter" relaxation times, the precursors lead to flow-induced crystalline structures (crosses). For intermediate times, i.e. in the transition zone (dashed area), there is still a few flow-induced crystalline structures near the fiber surface due to some remaining precursors. The images were recorded by polarized optical microscopy at $\mathrm{T}_{\mathrm{po}}=162.5^{\circ} \mathrm{C}$.

there is much less enhanced nucleation near the fiber surface due to partial relaxation of flow-induced precursors, which results in a significant reduction of oriented structures. These different behaviors are illustrated by POM images recorded at $\mathrm{T}_{\mathrm{po}}=162.5^{\circ} \mathrm{C}$. It should be noted that at higher pull-out temperature, the critical relaxation time $t_{c}{ }^{*}$ for precursors decay becomes shorter, in agreement with relaxation studies performed on other polymers. ${ }^{[12,14]}$ Extrapolating from the relaxation temperature map of PLA precursors given in Fig. 1, a pull-out temperature $T_{p o}=150^{\circ} \mathrm{C}$ is a judicious choice to obtain shear-induced precursors that do not significantly relax during cooling to the crystallization temperature $T_{c}=130{ }^{\circ} \mathrm{C}$, because they would need at least a few hours to noticeably relax. This is the reason why the fiber pulling procedure to obtain the highest density of shear-induced precursors was performed at $\mathrm{T}_{\text {po }}=150^{\circ} \mathrm{C}$ (protocol 2), with the purpose of examining the amorphous phase present within highly oriented structures and comparing its characteristics with the amorphous phase in conventional spherulites. The crystallization time $t_{c}=90$ min also ensured that the cylindrical structure is not embedded within a "sea" of spherulites, facilitating its isolation to perform calorimetric experiments. 


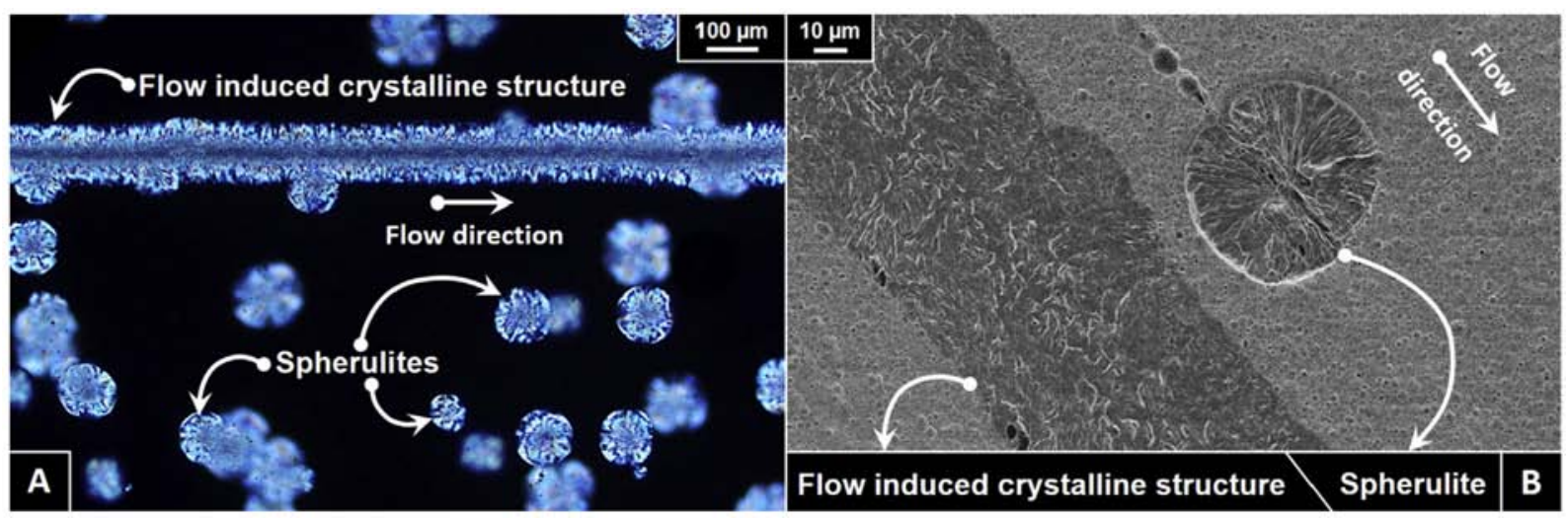

Fig. 2. Polarized optical $(A)$ and scanning electron $(B)$ micrographs of spherulites and flow-induced crystalline structures $\left(\mathrm{T}_{\mathrm{po}}=150^{\circ} \mathrm{C} ; \mathrm{t}^{*}<<\mathrm{t}_{\mathrm{c}}^{*} ; \mathrm{T}_{\mathrm{c}}=130{ }^{\circ} \mathrm{C} ; \mathrm{t}_{\mathrm{c}}=\right.$ $90 \mathrm{~min})$.

Fig. 2 shows POM and SEM pictures of semi-crystalline PLA subjected to protocol 2, in which the fiber is fully pulled out of the polymer melt. Fig. 2(A) shows that flow-induced precursors resulted in flow-induced crystalline structures, in the form of a cylindrical structure whose diameter reached approximately $100 \mu \mathrm{m}$ after a crystallization time $\mathrm{t}_{\mathrm{c}}=90$ min. The shear rate strongly decreases as the distance from the fiber increases, therefore no precursors were induced away from the pull-out region, and only spherulites were observed. Note that the diameter of the spherulites also reached approximately $100 \mu \mathrm{m}$. Fig. 2(B) showcases the differences between flow-induced crystalline structures and spherulites through the contrast between amorphous and crystalline areas: lamellar growth in cylindrical flow-induced crystalline structures mostly occurred in the direction perpendicular to the flow ${ }^{[31]}$ — that is to say that flow-induced crystalline structures are anisotropic, whereas spherulites developed radially.

The differences between flow-induced crystalline structures and spherulites, as well as their influence on the mobile amorphous fraction, were further investigated by FSC at the micro-scale, i.e. at the typical length-scale of semi-crystalline structures. Fig. 3 (A-B) shows cross sectional slices of both flow-induced crystalline structures and spherulites as obtained by ultramicrotomy prior to FSC analysis. In both slices, the semi-crystalline structure (dashed ellipse) is surrounded by an amorphous matrix. Two approaches were used to investigate the amorphous fraction. In the first approach, the whole sample (semi-crystalline structure along with surrounding amorphous matrix) was analyzed (SCA systems). In the second approach, only a portion of the semi-crystalline structure was isolated and analyzed, as indicated by the dashed polygon in Fig. 3(A-B) (SC systems). Fig. 3(C) shows a slice of flow-induced crystalline structures after FSC analysis. 


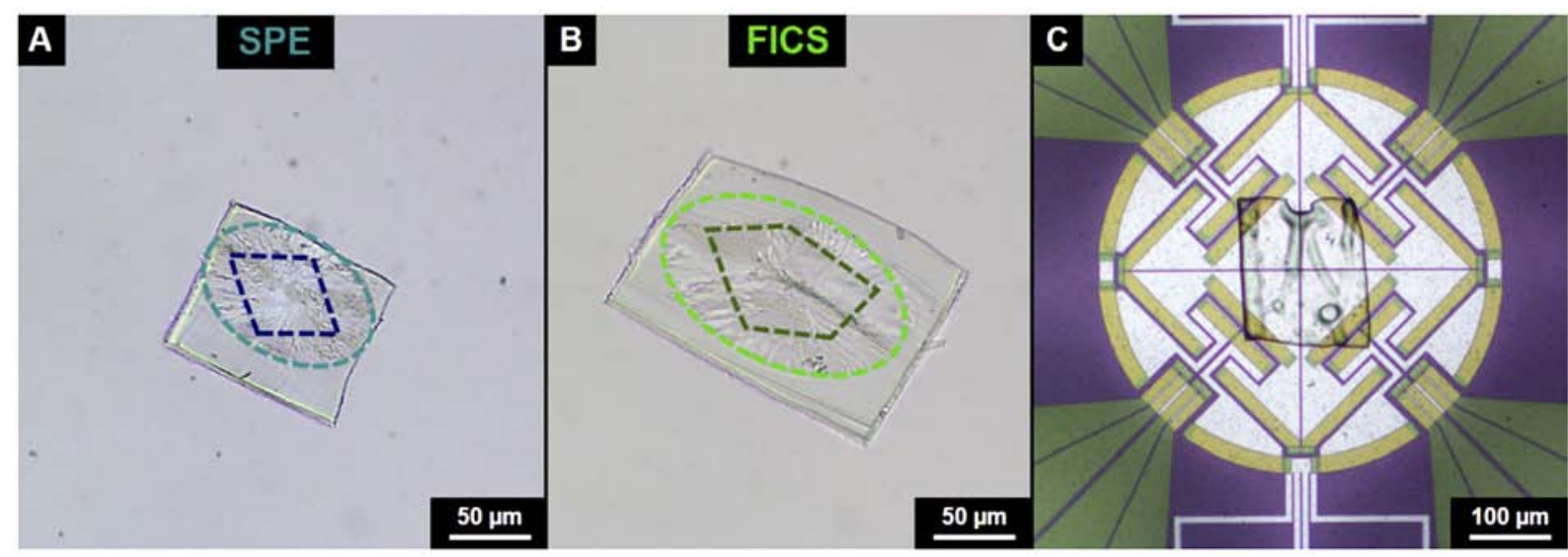

Fig. 3. Optical micrographs of slices of a spherulite (SPE) (A) and a flow-induced crystalline structure (B) obtained by ultramicrotomy prior to FSC analysis, as well as an example of a flow-induced crystalline structure slice placed on the FSC chip sensor after analysis (C). First, the sample within the ellipse plus the surrounding amorphous fraction were analyzed (SCA system), then a fresh slice was used to further isolate the dashed polygon within the semi-crystalline structures (SC system). The slices were $500 \mathrm{~nm}$ thick.
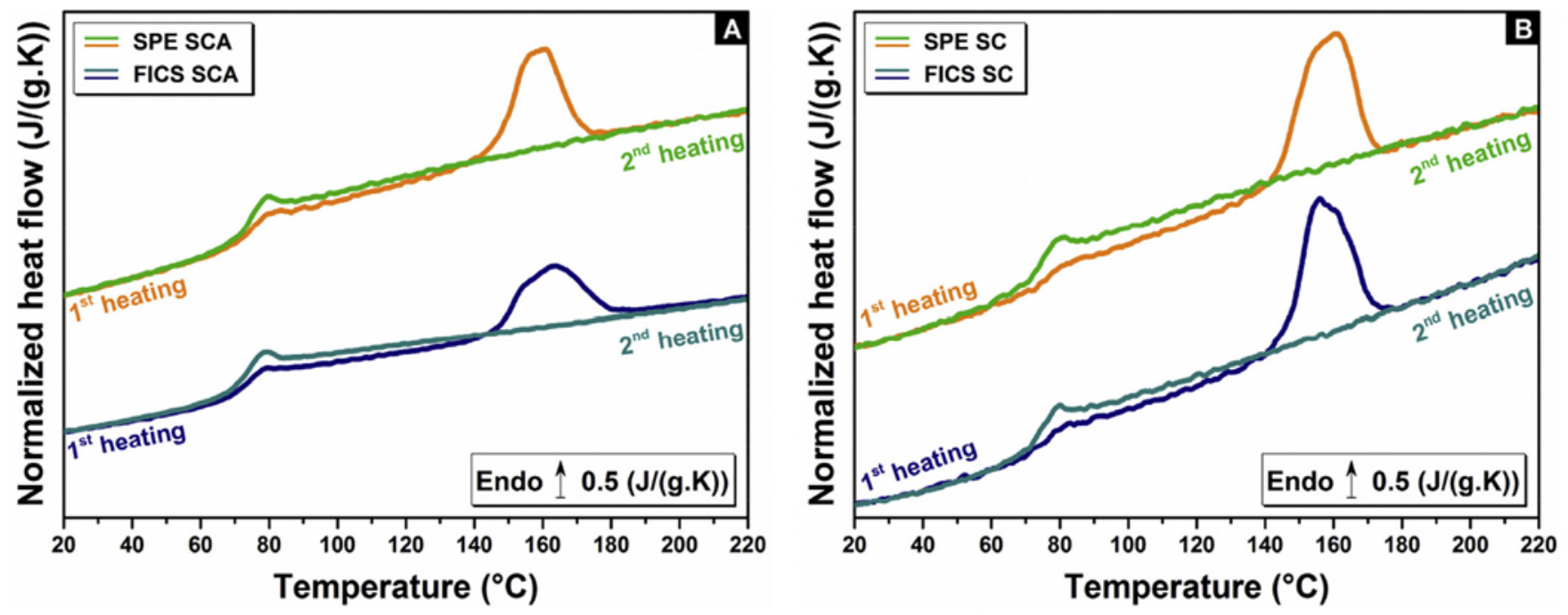

Fig. 4. FSC normalized heat flows of the first and second heating ramp from 20 ${ }^{\circ} \mathrm{C}$ to $220^{\circ} \mathrm{C}$ for spherulites (SPE) and flow-induced crystalline structures: (A) SCA vs. (B) SC systems. SPE = spherulite, FICS = flow-induced crystalline structure, SCA $=$ semi-crystalline structure and the surrounding amorphous phase, $\mathrm{SC}=$ semicrystalline structure.

Fig. 4 reports FSC heat flows normalized to the sample mass and the heating rate recorded during the first and second heating ramps from 20 ${ }^{\circ} \mathrm{C}$ to $220^{\circ} \mathrm{C}$ both for a slice of flow-induced crystalline structures and spherulites. Fig. 4(A) shows the results of SCA systems, whereas Fig. 4(B) 
shows the results of SC systems. For all systems, the first heating ramp includes two thermal events: an endothermic step typical of the glass transition within the amorphous fraction, followed by an endothermic event related to the melting of the crystalline fraction. The second heating ramp reveals that all the systems were perfectly quenched upon cooling $\left(\beta_{c}=\right.$ $1500 \mathrm{~K} \mathrm{~s}^{-1}$ ), as indicated by the increase in the magnitude of the heat flow step at the glass transition and the absence of the melting peak, that are typical of a fully amorphous material.

Melting occurs with a broad peak for both flow-induced crystalline structures and spherulites, either within SCA or SC systems. When PLA is crystallized at $130{ }^{\circ} \mathrm{C}$, only highly ordered $\alpha$ crystals are formed. ${ }^{[26] ~ I n ~ a d-~}$ dition, the use of high scanning rates $\left(1500 \mathrm{~K} \mathrm{~s}^{-1}\right)$ prevents structural reorganization of the crystals, which means that the broadening of the melting peaks is, in this case, only due to the complexity of the investigated semicrystalline structures. Table 1 summarizes the values of enthalpy associated to the melting peaks observed during the first heating ramps in Fig. 4 , along with the physical properties measured at the glass transition. The glass transition (measured at the mid-point) of completely amorphous PLA was found to be $70.9^{\circ} \mathrm{C}$, with a step in heat capacity of $0.51 \mathrm{~J} /(\mathrm{g} . \mathrm{K})$. This value was taken as a reference to estimate the mass of the sliced sample deposited onto the chip sensor, as described in Section 2.4. The mass of the sample was then used to correlate the step in heat capacity observed for the semicrystalline systems with their respective degree of crystallinity. The glass transition temperature of the SCA systems reaches $72.7^{\circ} \mathrm{C}$ and $72.9^{\circ} \mathrm{C}$ respectively, with a step of heat capacity that drops to $0.35 \mathrm{~J} /(\mathrm{g} . \mathrm{K})$ and 0.37 $\mathrm{J} /$ (g.K) for flow-induced crystalline structures and spherulites, respectively. The glass transition temperature of the SC systems reaches $74.2{ }^{\circ} \mathrm{C}$ and $74.1{ }^{\circ} \mathrm{C}$ respectively, with a step in heat capacity that drops to $0.32 \mathrm{~J} /(\mathrm{g} . \mathrm{K})$ and $0.30 \mathrm{~J} /(\mathrm{g} . \mathrm{K})$ for flow-induced crystalline structures and spherulites, respectively. Independently of the semi-crystalline morphology (flow-induced

Table 1. Thermal and microstructural properties measured while heating completely amorphous, flow-induced crystalline structures (FICS) and spherulites (SPE), both for SCA and SC systems: glass transition temperature $\mathrm{T}_{\text {gmid }}$, heat capacity step $\Delta \mathrm{C}_{\mathrm{p}^{\prime}}$ enthalpy of melting $\Delta \mathrm{H}_{\mathrm{m}^{\prime}}$ mobile amorphous fraction $\mathrm{X}_{\mathrm{MAF}^{\prime}}$ crystalline fraction $\mathrm{X}_{\mathrm{C}^{\prime}}$ and rigid amorphous fraction $\mathrm{X}_{\mathrm{RAF}}$.

\begin{tabular}{lllllll} 
& $\begin{array}{l}T_{\text {gmid }} \\
\left({ }^{\circ} \mathrm{C} \pm 1\right)\end{array}$ & $\begin{array}{l}\Delta C_{p} \\
(J /(g . K) \pm 0.05)\end{array}$ & $\begin{array}{l}\Delta H_{m} \\
(J / g \pm 3)\end{array}$ & $\begin{array}{l}X_{\text {MAF }} \\
(\% \pm 5)\end{array}$ & $\begin{array}{l}X_{c} \\
(\% \pm 5)\end{array}$ & $\begin{array}{l}X_{R A F} \\
(\% \pm 10)\end{array}$ \\
\hline Amorphous & 71 & 0.51 & 0 & 100 & 0 & 0 \\
SPE SCA & 73 & 0.35 & 24 & 69 & 18 & 13 \\
FICS SCA & 73 & 0.37 & 18 & 73 & 13 & 14 \\
SPE SC & 74 & 0.32 & 37 & 63 & 27 & 10 \\
FICS SC & 74 & 0.30 & 33 & 59 & 24 & 17 \\
\hline
\end{tabular}


crystalline structures and spherulites), the only parameter that differs is the amount of surrounding amorphous fraction, which explains the decrease in the step of heat capacity when comparing SCA and SC systems. In addition, the increase of the mid-point glass transition temperature of the SCA and SC systems with respect to completely amorphous PLA $\left(2{ }^{\circ} \mathrm{C}\right.$ and $3{ }^{\circ} \mathrm{C}$ respectively) is due to the steric hindrances generated by the presence of the crystalline fraction. This is why intra-spherulitic amorphous phase is called constrained MAF. The glass transition of SCA systems is halfway between the glass transition temperature of the amorphous and SC systems due to the presence of both inter-spherulitic and intraspherulitic regions. From the enthalpy of melting reported in Table 1, the crystalline fraction $X_{c}$ was determined according to the following equation:

$$
X_{c}=\frac{\Delta H_{m}}{\Delta H_{m}^{0}}
$$

in which $\Delta \mathrm{H}_{\mathrm{m}}$ is the enthalpy of melting and $\Delta \mathrm{H}_{\mathrm{m}}^{0}$ is the enthalpy of melting of a $100 \%$ crystallized PLA $(136 \mathrm{~J} / \mathrm{g}$ ) which was obtained using the recent formula proposed by Righetti et al. ${ }^{[59]}$ From the crystalline fraction $X_{c}$ and the step of heat capacity at the glass transition $\Delta C_{p^{\prime}}$ the mobile amorphous fraction $\mathrm{X}_{\mathrm{MAF}}$ and the rigid amorphous fraction $\mathrm{X}_{\mathrm{RAF}}$ were estimated according to the following equations:

$$
\begin{gathered}
\mathrm{X}_{\mathrm{MAF}}=\frac{\Delta \mathrm{C}_{\mathrm{p}}}{\Delta \mathrm{C}_{\mathrm{p}}^{0}} \\
1=\mathrm{X}_{\mathrm{MAF}}+\mathrm{X}_{\mathrm{RAF}}+\mathrm{X}_{\mathrm{C}}
\end{gathered}
$$

in which $\Delta C_{p}^{0}=0.51 \mathrm{~J} /(\mathrm{g} . \mathrm{K})^{[58]}$ is the heat capacity step of completely amorphous PLA. The values are summarized in Table 1. By comparing flow-induced crystalline structures or spherulites from SCA on one side or SC systems on the other side, it is interesting to observe that flow-induced crystalline structures or spherulites approaches have no influence on the apportionment of microstructural fractions. Nevertheless, SCA systems develop $\mathrm{X}_{\mathrm{MAF}} \approx 70 \%$ and $\mathrm{X}_{\mathrm{C}} \approx 15 \%$, whereas SC systems rather develop $\mathrm{X}_{\mathrm{MAF}}$ $\approx 60 \%$ and $X_{C} \approx 25 \%$, on average. Therefore, independently of the approach used to investigate the microstructure, an average value of $\mathrm{X}_{\mathrm{RAF}} \approx$ $15 \%$ was found. Apparently, the structural anisotropy induced in the liquid like-state plays no role on the apportionment of the different fractions. As observed by Righetti et al., ${ }^{[43,50]}$ the establishment of the RAF depends on the crystallization temperature, i.e. on the molecular mobility of the polymer chains. These results show that the shear conditions imposed prior to crystallization only affected the crystallization kinetics and the morphology 
of the final crystalline structure, with no influence on the amount of the RAF (which appears to be influenced only by the temperature conditions).

\subsection{Cooperative motions in amorphous and crystallized PLA at the glass transition}

Prior to FSC analysis, a visual estimation of the main microstructural areas developed in SCA systems was performed by optical microscopy. The ratio of amorphous to semi-crystalline surface areas was evaluated for both morphologies (flow-induced crystalline structures and spherulites) from the POM images shown in Fig. 3 (A-B). Table 2 reports the fraction of surrounding amorphous matrix, namely unconstrained MAF, for flow-induced crystalline structures and spherulites samples (35\% and $50 \%$ respectively). However, MAF $\left(\mathrm{X}_{\mathrm{MAF}}\right)$ measured by FSC, reach $69 \%$ and $73 \%$ for flow-induced crystalline structures and spherulites, respectively. Thus, from POM observations and FSC analysis, a discrepancy of $34 \%$ and $23 \%$ in the amorphous fractions was evidenced between flow-induced crystalline structures and spherulites. This discrepancy is related to the fraction of constrained MAF $\left(\mathrm{X}_{\mathrm{CMAF}}\right)$ within flow-induced crystalline structures and spherulites, as reported in Table 2. In the few past years, the existence of a so-called intraspherulitic MAF has been shown for semi-crystalline polyesters such as PET and PLA, ${ }^{[52,54,55,57,71]}$ which behaves like a constrained MAF. Wang et al. ${ }^{[52]}$ showed that semi-crystalline PLA with different crystallinity degrees has different glass transition dynamics after structural relaxation. In particular, the process occurring at lower temperature was attributed to the bulk-like glass transition, whereas the process occurring at higher temperature was attributed to the glass transition of constrained MAF, i.e. the amorphous fractions whose motions were hindered by the lamellar stacks. Later on, Delpouve et al. ${ }^{[54]}$ investigated the relaxation dynamics of inter- and intraspherulitic amorphous phases through the concept of CRR according to the thermodynamic approach proposed by Donth. The characteristic value

Table 2. Visual estimation of amorphous and semi-crystalline sample areas on the slices of spherulites (SPE) and flow-induced crystalline structures (FICS) in Fig. 3 (A-B). Prior to thermal analysis, the total sample area $A_{\text {tot' }}$ the semi-crystalline sample area $A_{\text {sc }}$ as well as the ratio of amorphous (A matrix) to semi-crystalline (SC structure) sample areas of the slice have been estimated by picture analysis. They highlight the unconstrained $\left(X_{\mathrm{UCMAF}}\right)$ and the constrained $\left(\mathrm{X}_{\mathrm{CMAF}}\right)$ fractions representing the mobile amorphous fraction $\left(\mathrm{X}_{\mathrm{MAF}}\right)$.

\begin{tabular}{llllll}
$\begin{array}{l}A_{\text {tot }} \\
\left(\mu m^{2}\right)\end{array}$ & $\begin{array}{l}A_{\text {sc }} \\
\left(\mu m^{2}\right)\end{array}$ & $\begin{array}{l}\text { A matrix } \\
(\%)\end{array}$ & $\begin{array}{l}\text { SC structure } \\
(\%)\end{array}$ & $\begin{array}{c}X_{\text {MAF }}=X_{\text {CMAF }} \\
+X_{\text {UCMAF }} \\
(\% \pm 5)\end{array}$ \\
\hline SPE & $10500 \pm 300$ & $6800 \pm 100$ & $35 \pm 5$ & $65 \pm 5$ & $69=34+35$ \\
FICS & $21400 \pm 700$ & $10800 \pm 200$ & $50 \pm 5$ & $50 \pm 5$ & $73=23+50$ \\
\hline
\end{tabular}


of the cooperativity length at the glass transition $(3.0 \mathrm{~nm}$ for a completely amorphous PLA) was found to decrease to $2.0 \mathrm{~nm}$ when the maximum degree of crystallinity was reached. For intermediate degrees of crystallinity, the cooperativity length associated to the dynamics of both amorphous fractions was observed to decrease linearly from $3.0 \mathrm{~nm}$ to $2.0 \mathrm{~nm}$. It turned out that cooperativity could be correlated to the structural relaxation of the considered fractions. ${ }^{[53]}$ By tracking the temperature evolution of the peaks associated to the enthalpy recovery in amorphous, partially crystallized and fully crystallized systems, they discussed the molecular dynamics of both inter- and intra-spherulitic amorphous phases, and found it in agreement with the results previously published by Wang et al. ${ }^{[52]}$ In particular for partially crystallized systems depicting double peaks, the enthalpy recovery of the inter-spherulitic amorphous fraction was found to be similar to the one observed for a completely amorphous sample, whereas the enthalpy recovery of the intraspherulitic amorphous fraction was found to depend on degree of crystallinity. A system that is partially crystallized would show an enthalpy recovery peak related to intra-spherulitic amorphous fraction whose maximum appears at lower temperatures with respect to the same system crystallized to the maximum extent. The temperature shift was attributed to the differences induced by the crystallization process, that is to say secondary crystallization and the development of RAF. $^{[43]}$ In literature it has been shown that a decrease in cooperativity can be associated to a decrease in the thickness of the amorphous fraction confined within the crystal[ ${ }^{[60]}$, as well as to isochoric ${ }^{[61]}$ and multilayer ${ }^{[62]}$ confinement. Thus, the molecular dynamics of the constrained MAF depends on the lamellar thickness.

In addition to the aforementioned glass transition properties, the glass transition process was also evaluated in terms of CRR. From modulated temperature DSC, the cooperativity volume $\xi_{\mathrm{T} \alpha}{ }^{3}$ at the dynamic glass transition temperature $T_{\alpha}$ is extracted from the following equation:

$$
\xi_{T_{\alpha}}^{3}=\frac{\left(1 / C_{p}\right)_{\text {Glass }}-\left(1 / C_{p}\right)_{\text {Liquid }}}{\rho(\delta T)^{2}} \mathrm{k}_{\mathrm{B}} T_{\alpha}^{2}
$$

with $\mathrm{k}_{\mathrm{B}}$ the Boltzmann constant, $\delta \mathrm{T}$ the average temperature fluctuation related to the dynamic glass transition of a CRR, $\rho$ the density of the relaxing system, and $C_{p}$ the heat capacity at constant pressure. From DSC, or in this work FSC, the dynamic glass transition temperature $T_{\alpha}$ corresponds to the glass transition temperature $T_{g^{\prime}}$ whereas the average temperature fluctuation $\delta T$ is estimated upon heating from the following equation: ${ }^{63]}$

$$
\delta T=\Delta T / 2.5
$$


with $\Delta T$ the temperature interval where the $C_{p}$ curve from FSC varies between $16 \%$ and $84 \%$ of the heat capacity step $\Delta C_{p}$. This rule has been checked for several substances in literature. ${ }^{[63]}$ The different experimental parameters necessary to estimate the cooperativity volume $\xi_{T \alpha}{ }^{3}$ are summarized in Table 3. In order to assess the molecular dynamics of constrained MAF, only SC systems were investigated. Such an approach cannot be used in the case of SCA systems because an average glass transition would be measured including both constrained MAF and the surrounding amorphous matrix (unconstrained MAF). With the values of glass transition temperature and heat capacity step reported above, similar values of mean fluctuation temperature, cooperativity length and volume were obtained for both flow-induced crystalline structures and spherulites. However, the mean fluctuation temperature at the glass transition for constrained MAF in flow-induced crystalline structures and spherulites is higher compared to the value obtained for completely amorphous PLA (5.4 K vs. 3.8 K). As a consequence, the cooperativity length and volume of the amorphous chains trapped within semi-crystalline structures is reduced to $1.9 \mathrm{~nm}$ and $7 \mathrm{~nm}^{3}$ respectively, as compared to the values of $2.6 \mathrm{~nm}$ and $18 \mathrm{~nm}_{3}$ that are classically observed for completely amorphous PLA. ${ }^{[3,58]}$ These results are consistent with the reduction of mobility induced by the presence of lamellar stacks already reported by Wang et al. ${ }^{[52]}$ and Delpouve et al. ${ }^{[54]}$ Independently of the SC system, CRR are $30 \%$ smaller with respect to completely amorphous PLA. This result suggests that constrained MAF behaves the same independently of the type of semicrystalline morphology. Once again, the molecular dynamics of constrained MAF seems to be driven by the crystallization temperature, and therefore the competition between crystallization and vitrification, rather than by the crystalline morphology. However, another parameter should be taken into account: the degree of connection of the crystalline fraction to the surrounding amorphous fraction, i.e. the decoupling of fractions and the development of the RAF.

Table 3. Parameters obtained by FSC and used to calculate the size of the Cooperative Rearranging Regions (CRR) for completely amorphous and semi-crystalline PLA, in the case of SC systems only, for both spherulite (SPE) and flow-induced crystalline structures (FICS): mean temperature fluctuation $\delta \mathrm{T}$, cooperativity length $\xi_{\mathrm{T \alpha}}$ and cooperativity volume $\xi_{\mathrm{T} \alpha}{ }^{3}$.

\begin{tabular}{lccc} 
& $\delta \mathrm{T}(\mathrm{K})$ & $\xi_{\mathrm{T \alpha}}(\mathrm{nm})$ & $\xi_{\mathrm{T \alpha}} 3\left(\mathrm{~nm}^{3}\right)$ \\
\hline Amorphous & $3.8 \pm 0.3$ & $2.6 \pm 0.3$ & $18 \pm 2$ \\
SPE SC & $5.4 \pm 0.5$ & $1.9 \pm 0.2$ & $7 \pm 1$ \\
FICS SC & $5.3 \pm 0.5$ & $1.9 \pm 0.2$ & $7 \pm 1$ \\
\hline
\end{tabular}




\subsection{Local motions in amorphous and crystallized PLA below the glass transition}

The molecular mobility of the amorphous fractions through the wellknown phenomenon of structural relaxation and physical aging was then investigated. Using physical aging to reveal differences in molecular dynamics by thermal analysis is a common procedure. ${ }^{[52,54]}$ In addition, the high scanning rates attainable by FSC allow accelerating the kinetics of physical aging, because glassy states with higher level of enthalpy can be formed. FSC allowed performing physical aging on a wider range of time (from milliseconds to days), reaching thermodynamic equilibrium. ${ }^{[40]}$

Fig. 5 shows FSC normalized heat flows for aged PLA. Fig. 5(A) focuses on completely amorphous PLA. As aging progresses, a sequence of endothermic peaks is classically observed indicating the structural relaxation process superimposed to the glass transition. Besides, the endothermic peaks increase and shift towards high temperature as the aging time increases. Physical aging of SCA systems is more complex, as shown in Fig. $5(B-C)$. The peaks of enthalpy recovery for both flow-induced crystalline structures and spherulites are clearly divided into two contributions that become more and more visible as aging time increases, indicating that a different behavior is observed for a crystalline environment. A similar behavior has already been reported in the literature for PLA as previously detailed above, ${ }^{[53,55]}$ explained by the existence of two amorphous fractions having their own response to structural relaxation due to their respective environment. It has also been suggested that the high-temperature contribution could be related to the enthalpy recovery of constrained MAF, whereas the low temperature contribution would be associated to the relaxation of unconstrained MAF, i.e. the surrounding amorphous matrix.

When the SC systems are considered, only one endothermic peak for both flow-induced crystalline structures and spherulites is observed. In comparison with amorphous PLA, the enthalpy recovery peaks are broadened and shifted towards higher temperatures within a larger range of temperatures, as shown in Fig. 5 (D-E). This result could be due to the strong influence of the semi-crystalline environment on the molecular dynamics of the confined amorphous fraction, suggesting a stronger degree of confinement induced by tighter inter-lamellar stacks. ${ }^{[53]}$

First of all, Fig. 6(A) shows the Gaussian fitting procedure used to track the maximum $T_{p}$ of the enthalpy recovery peak for all the contributions observed in Fig. 5. The Gaussian fit was performed on the normalized heat flow subtraction (aged - rejuvenated), as shown through the example of flow-induced crystalline structures SCA aged 1 min. Fig. 6(B) reports the temperature evolution of the maximum of the enthalpy recovery peak as 
Fig. 5. Normalized heat flows recorded after physical aging of completely amorphous PLA (A), SPE SCA (B), FICS SCA (C), SPE SC (D) and FICS SC (E). Aging was performed in situ by FSC at a scanning rate of $I \beta_{c} \mid=\beta_{h}=1500 \mathrm{~K}$ $\mathrm{s}^{-1}$ at $\mathrm{T}_{\mathrm{ag}}=\mathrm{T}_{\mathrm{g} \text {,cooling }}-9^{\circ} \mathrm{C}=58^{\circ} \mathrm{C}$ for aging times ranging from $0.001 \mathrm{~min}$ to $10 \mathrm{~min}$. Glass transition temperature measured upon cooling are displayed in supporting information. SPE = spherulite, FICS = flow-induced crystalline structure, SCA = semi-crystalline structure and the surrounding amorphous phase, $\mathrm{SC}=$ semicrystalline structure.
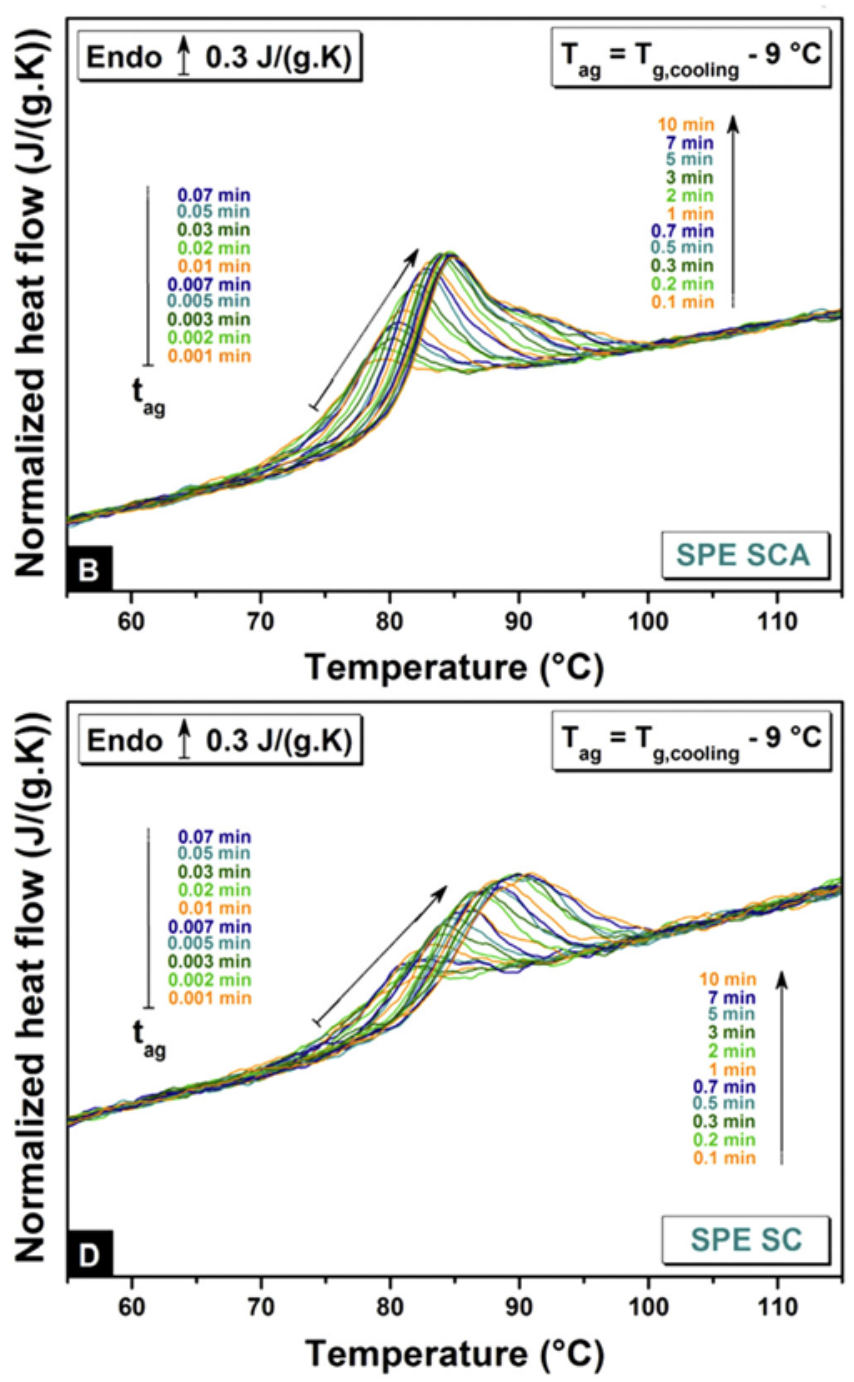
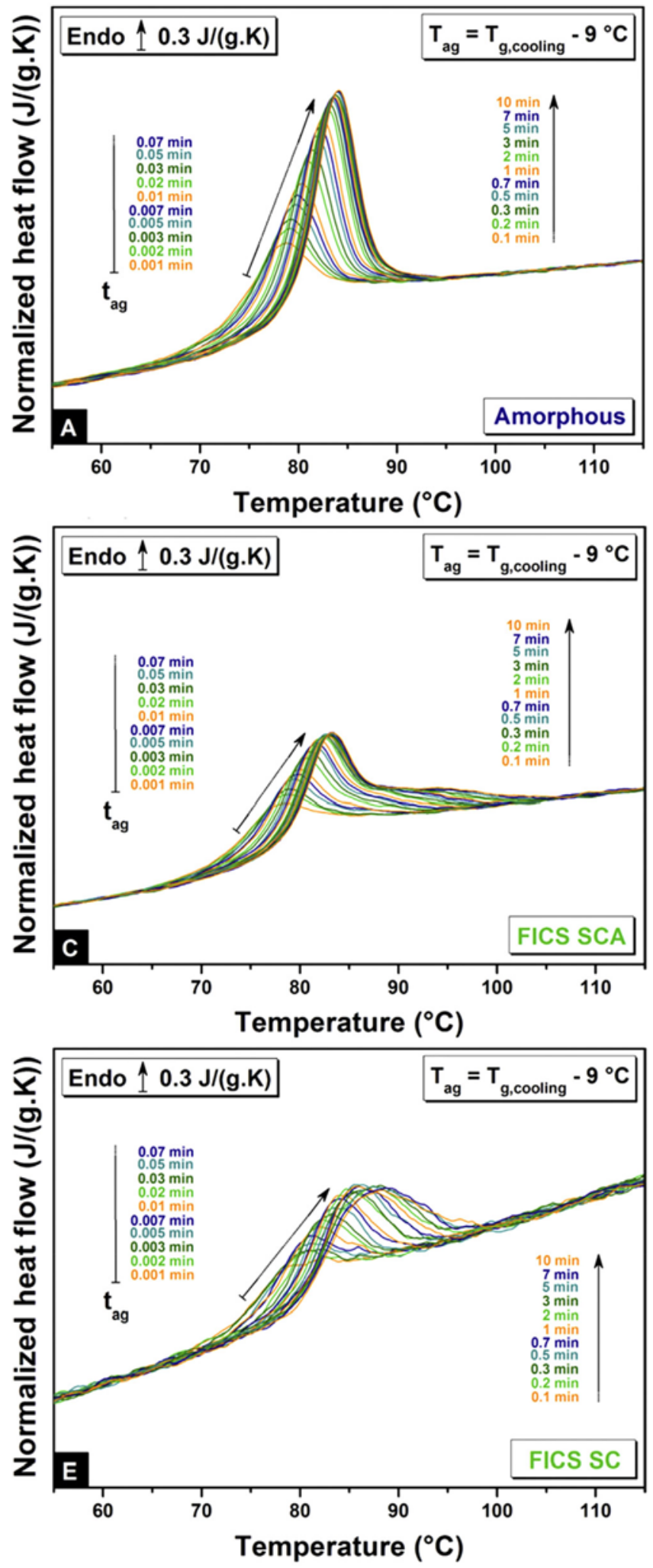

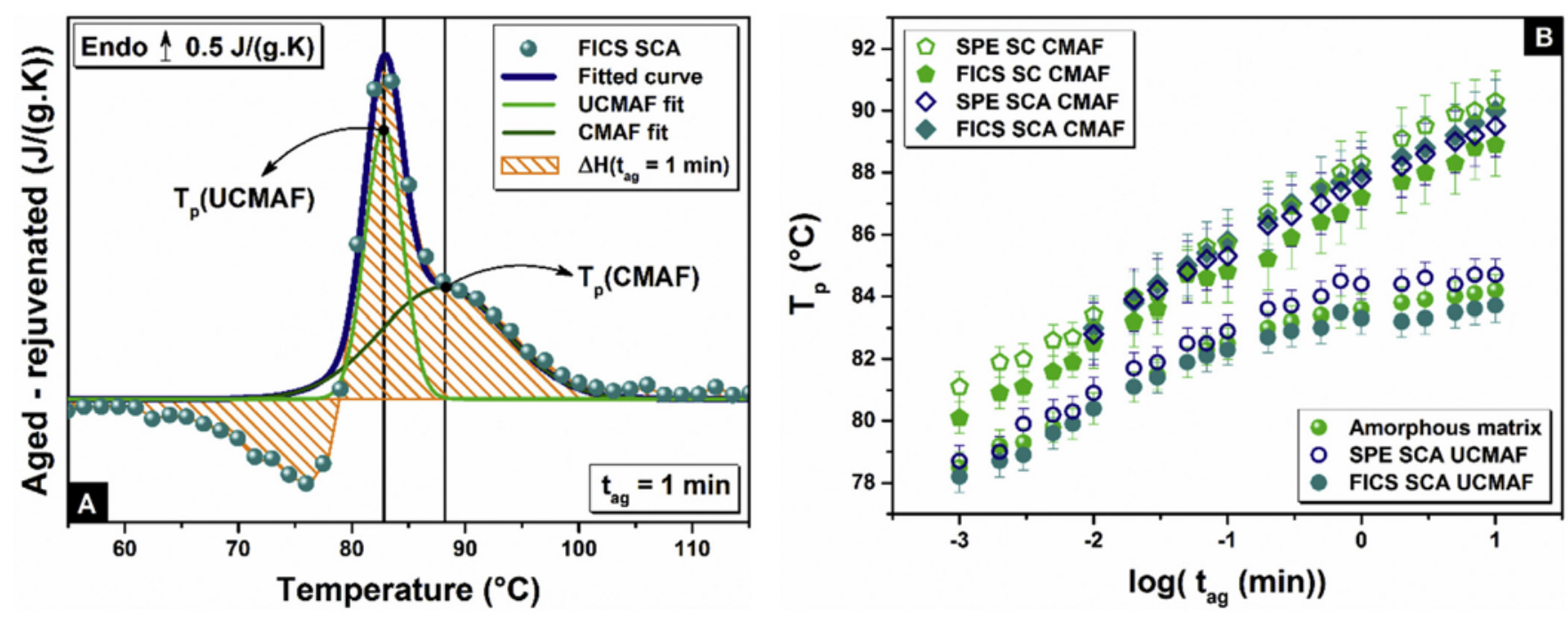

Fig. 6. (A) Normalized heat flow subtraction (aged - rejuvenated) of FICS SCA aged 1 min $9^{\circ} \mathrm{C}$ below $T_{g}$, cooling. The curve illustrates how the enthalpy recovery $\Delta H_{\text {tag }}$ was calculated, as well as the fitting procedure for the maximum $T_{p}$ of the enthalpy recovery peak. (B) Evolution of the maximum $T_{p}$ of the enthalpy recovery peak as a function of the aging time. Spheres: completely amorphous PLA. Discs: unconstrained mobile amorphous fraction (UCMAF) within the SCA systems (FICS: filled discs, SPE: empty discs). Diamonds: constrained mobile amorphous fraction (CMAF) within the SCA systems (SPE: empty diamonds, FICS: filled diamonds). Pentagons: constrained mobile amorphous fraction (CMAF) within the SC systems (SPE: empty pentagons, FICS: filled pentagons). SPE = spherulite, FICS = flow-induced crystalline structure, $\mathrm{SCA}=$ semi-crystalline structure and the surrounding amorphous phase, $\mathrm{SC}=$ semi-crystalline structure.

a function of aging time. Regarding the SCA systems, the first contribution of both flow-induced crystalline structures and spherulites behaves as unconstrained MAF in that a linear increase is observed until a plateau is reached at about $84^{\circ} \mathrm{C}$ after the same aging duration. This means that the first contribution recorded for both flow-induced crystalline structures and spherulites is actually related to the surrounding amorphous matrix. As for the second contribution, SCA and SC systems show no differences: the trend is the same independently of the type of semi-crystalline morphology, and is shifted to higher temperatures as compared to the amorphous matrix. Thus, in both systems, the second contribution seems to be related to the amorphous fraction constrained within the lamellar stacks, with the same molecular dynamics.

Then, as graphically illustrated in Fig. 6(A) by the orange hatched area, the enthalpy recovery of a glass aged at a temperature $T_{a g}$ during a time $t_{a g}$ 
is calculated by integrating the difference between the scans of the aged and rejuvenated sample, according to the following equation:

$$
\Delta H=\left(T_{a g^{\prime}} t_{a g}\right)=\int_{T_{1}}^{T_{2}}\left[C_{p}^{a}(T)-C_{p}^{r}(T)\right] \cdot d T
$$

in which $C_{p}^{a}(T)$ and $C_{p}{ }^{r}(T)$ are the specific heat of the aged and rejuvenated samples respectively, and $T_{1}$ and $T_{2}$ are arbitrary temperatures below and above the glass transition temperature $\mathrm{T}_{9}{ }^{\left[{ }^{[64]}\right.}$ The so-called enthalpy recovery represents the energy required by the glass to reach its thermodynamic equilibrium. Under the assumption that equilibrium is reached for an infinite time, the expected total enthalpy loss $\Delta \mathrm{H}_{\infty}$ extrapolated from the equilibrium melt depends on the aging temperature $T_{a g^{\prime}}$ as well as on the glass transition temperature $T_{g}$ and the heat capacity step $\Delta C_{p}$ of the sample, according to the following relation:

$$
\Delta H_{\infty}=\Delta C_{p} \cdot\left(T_{g}-T_{a g}\right)
$$

Fig. 7(A) shows the evolution of the difference between the enthalpy recovery $\Delta \mathrm{H}_{\text {tag }}$ and the total enthalpy loss $\Delta \mathrm{H}_{\infty}$ as a function of aging time $\mathrm{t}_{\mathrm{ag}}$ for a completely amorphous PLA, as well as for the semi-crystalline samples
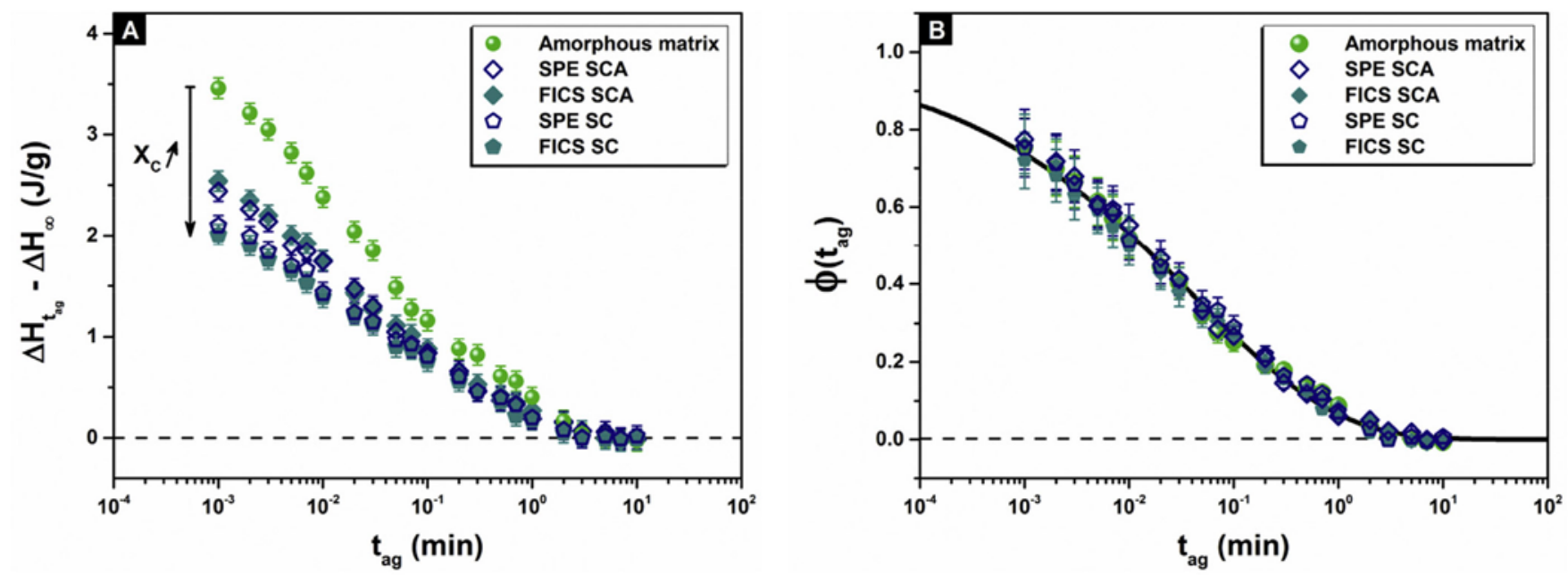

Fig. 7. Time evolution of the difference between the enthalpy recovery $\Delta \mathrm{H}_{\text {tag }}$ and the total enthalpy loss $\Delta \mathrm{H}_{\infty}(\mathrm{A})$, and of the structural relaxation function $\varphi$ (B). Spheres: completely amorphous PLA. Diamonds: SCA systems. Pentagons: SC systems. Empty symbols: SPE. Filled symbols: FICS. SCA = semi-crystalline structure and the surrounding amorphous phase, $\mathrm{SC}=$ semi-crystalline structure, $\mathrm{SPE}=$ spherulite, FICS = flow-induced crystalline structure. Black line is a guide for eyes. 
(both flow-induced crystalline structures and spherulites morphologies, as well as SCA and SC systems). When the crystallinity degree increases, the amorphous response decreases (Table 1). Therefore the enthalpy recovery and the total enthalpy loss values are smaller for the semi-crystalline systems with respect to the completely amorphous sample due to the decrease of MAF. To compare the kinetics and the dynamics, the structural relaxation function $\varphi$ has been plotted as a function of the aging time. The structural relaxation function $\varphi$ is defined as:

$$
\varphi\left(t_{a g}\right)=\frac{\Delta H_{t_{a g}}-\Delta H_{\infty}}{\Delta H_{\infty}}
$$

in which $\Delta \mathrm{H}_{\mathrm{t}_{\mathrm{ag}}}$ and $\Delta \mathrm{H}_{\infty}$ are the enthalpy recovery after an aging time $\mathrm{t}_{\mathrm{ag}}$ and the total enthalpy loss after an infinite aging time, respectively. This function allows normalizing the enthalpy recovery to the same amount of MAF. Fig. 7(B) shows that the evolution of enthalpy recovery is the same independently of the environment of the MAF (both type of morphology and degree of crystallinity). Besides, the whole structural relaxation process has been accelerated by the high scanning rates available in FSC ${ }^{[40]}$ : all the samples reached the thermodynamic equilibrium after the same aging duration. It is worth mentioning that all systems superimpose, and depict the same physical aging kinetics. Therefore, the structural relaxation of the amorphous fraction depends neither on the microstructure, on the coupling between fractions, on the confinement by the crystals nor on the orientation of the macromolecules prior to crystallization.

Physical aging is performed at a given temperature below the glass transition. During sub- $T_{g}$ structural relaxation far from the glass transition, only local molecular motions, such as the rotation of sub-segmental flexible groups along the polymer backbone take place ${ }^{[65]}$. For example, Priestley et al. ${ }^{66]}$ have shown by dielectric studies a significant decrease in the strength of the $\beta$ relaxation dynamics when silica nanospheres are dispersed in a poly (methyl methacrylate) (PMMA) matrix. Such a decrease has been attributed to the hydrogen bonds between the flexible ester side-groups on PMMA and the hydroxyl groups on silica. Due to presence of silica, the hydrogen bonds inhibit the motions of the ester side-groups. Therefore, by assuming that the motions of the side-groups are the driving forces for PMMA structural relaxation, the reduction of physical aging rates far below $\mathrm{T}_{g}\left(\mathrm{~T}_{\mathrm{g}}-88^{\circ} \mathrm{C}\right)$ in silica-PMMA nanocomposites have been explained and associated to a reduction of the $\beta$ process. More recently, Cangialosi et al. ${ }^{[67]}$ observed two equilibration mechanisms during physical aging of polystyrene and polycarbonate. They reported either a single or a double decay in the enthalpy recovery, when physical aging is performed close to 
or far below the glass transition, respectively. The first (faster) decay has been assigned to secondary relaxations, whereas the second (slower) decay has been attributed to the $\alpha$ process. At the same time, Grenet et al. ${ }^{[68]}$ reported experimental results that were collected on glassy Selenium both after short-term and long-term aging, which support the idea that at least two separate mechanisms of relaxation coexist in physical aging, one with very fast kinetics and the other with much slower kinetics. Therefore, according to the aging temperature (close or far to the glass transition) local motions and/or cooperative motions might be involved in the physical aging process.

In this work, differences in the structural relaxation were observed by heating the samples through the glass transition, i.e. by measuring the results of cooperative motions during equilibrium recovery. Thus, the influence of the crystalline environment was observed on the macromolecular scale. However, no influence was highlighted during the structural relaxation, because only a single decay with the same kinetics for all systems was observed. Therefore, physical aging is assumed to be mainly driven by local motions in these aging conditions $\left(T_{a g}=T_{g, c o o l i n g}-9^{\circ} \mathrm{C}\right)$. Besides, this result suggests that contrarily to $\alpha$ relaxation, secondary relaxations are not influenced by the crystalline environment. It is worth mentioning that Schick et al. ${ }^{[70]}$ observed just a slight influence of the crystals on the local movements of MAF in the case of semicrystalline PET by dielectric measurements. Later on, Kattan et al. showed no-dependency of the $\beta$ relaxations with the degree of crystallinity on various amorphous and semi-crystalline polyesters, by thermally stimulated depolarization current experiments. ${ }^{[71]}$

To summarize, similar values of the enthalpy recovery normalized to the MAF were obtained for all the systems; however, the shape of the enthalpy recovery peaks was not the same. Amorphous PLA has a cooperativity length of $2.6 \mathrm{~nm}$ and sharper enthalpy recovery peaks because the relatively large cooperativity domains ease the recovery to the equilibrium upon heating. Consistently, SC PLA (both flow-induced crystalline structures and spherulites) have a reduced cooperativity length $(1.9 \mathrm{~nm})$ and broader enthalpy recovery peaks because the relatively small cooperativity domains delay the recovery to the equilibrium upon heating. To assess the nature of the molecular dynamics involved in the structural relaxation, between local or cooperative motions, further physical aging investigations with different aging temperatures (more or less close to the glass transition) should be performed. 


\section{Conclusion}

The relaxation of shear-induced precursors was investigated at high temperature to mimic the industrial injection process of PLA. At $150{ }^{\circ} \mathrm{C}$, the critical relaxation time is much larger than the time required to quench the sample to a suitable crystallization temperature, resulting in the formation of highly oriented cylindrical structures which are embedded in an amorphous PLA matrix. This observation allowed investigating the influence of the crystalline environment generated either by flow-induced crystallization or quiescent crystallization on the amorphous phase after a thermal treatment performed in optimized conditions. Slices of the semicrystalline samples were analyzed by FSC to investigate the behavior of the amorphous phase at the same scale of the semicrystalline structure. Thanks to the high scanning rates accessible via FSC, physical aging was accelerated, which allowed scanning up to four decades of aging time. Segmental and local relaxations were investigated through the concept of cooperativity and physical aging, respectively. A decrease of the cooperativity length in the semi-crystalline systems was observed in comparison to completely amorphous PLA, essentially due to the coupling between phases and the confinement effect of the crystals. The molecular dynamics were observed to be the same independently of the semi-crystalline morphology. The molecular dynamics were found to depend on the crystallinity degree at the macromolecular scale. However, the same enthalpy recovery was measured for all the systems, meaning that the structural relaxation is not influenced by the crystallinity degree. This result is consistent with the fact that physical aging is mainly due to local motions, and local motions are not affected by the crystalline environment (different length-scale) at this aging temperature close to the glass transition. The only influence that could be observed is the one measured during the subsequent heating ramp through the $\alpha$ relaxation, i.e. the glass transition. Further physical aging investigations with different aging temperatures should be performed to assess the nature of the molecular dynamics involved during the physical aging.

Acknowledgments - The authors are grateful to the Normandy Region, France, for the financial support for PhD thesis and FSC equipment.

Appendix A. Supplementary data follows the References. 


\section{References}

1. S.Z.D. Cheng, B. Lotz, Enthalpic and entropic origins of nucleation barriers during polymer crystallization: the Hoffman-Lauritzen theory and beyond, Polymer 46 (2005) 8662-8681. doi 10.1016/j.polymer.2005.03.125

2. X.F. Lu, J.N. Hay, Isothermal crystallization kinetics and melting behaviour of poly(ethylene terephthalate), Polymer 42 (2001) 9423-9431. doi 10.1016/ S0032-3861(01)00502-X

3. H. Li, M.A. Huneault, Effect of nucleation and plasticization on the crystallization of poly(lactic acid), Polymer 48 (2007) 6855-6866. doi 10.1016/j.polymer.2007.09.020

4. H. Bai, C. Huang, H. Xiu, Q. Zhang, Q. Fu, Enhancing mechanical performance of polylactide by tailoring crystal morphology and lamellae orientation with the aid of nucleating agent, Polymer 55 (2014) 6924-6934. doi 10.1016/j. polymer.2014.10.059

5. B.O. Reid, M. Vadlamudi, A. Mamun, H. Janani, H. Gao, W. Hu, R.G. Alamo, Strong memory effect of crystallization above the equilibrium melting point of random copolymers, Macromolecules 46 (2013) 6485-6497. doi 10.1021/ ma400839d

6. D. Cavallo, L. Gardella, G. Portale, A.J. Müller, G.C. Alfonso, On cross- and selfnucleation in seeded crystallization of isotactic poly(1-butene), Polymer 54 (2013) 4637-4644. doi 10.1016/j.polymer.2013.06.051

7. D. Cavallo, L. Gardella, G. Portale, A.J. Müller, G.C. Alfonso, Kinetics of crossnucleation in isotactic poly(1-butene), Macromolecules 47 (2014) 870-873. doi 10.1021/ma402396f

8. Z. Wang, Z. Ma, L. Li, Flow-induced crystallization of polymers: molecular and thermodynamic considerations, Macromolecules 49 (2016) 1505-1517. doi 10.1021/acs.macromol.5b02688

9. F. Azzurri, G.C. Alfonso, Lifetime of shear-induced crystal nucleation precursors, Macromolecules 38 (2005) 1723-1728. doi 10.1021/ma0485989

10. R.H. Somani, L. Yang, L. Zhu, B.S. Hsiao, Flow-induced shish-kebab precursor structures in entangled polymer melts, Polymer 46 (2005) 8587-8623. doi 10.1016/j.polymer.2005.06.034

11. F. Azzurri, G.C. Alfonso, Insights into formation and relaxation of shearinduced nucleation precursors in isotactic polystyrene, Macromolecules 41 (2008) 1377-1383. doi 10.1021/ma071475e

12. Y. Hayashi, G. Matsuba, Y. Zhao, K. Nishida, T. Kanaya, Precursor of shishkebab in isotactic polystyrene under shear flow, Polymer 50 (2009) 20952103. doi 10.1016/j.polymer.2009.03.008

13. S. Kimata, T. Sakurai, Y. Nozue, T. Kasahara, N. Yamaguchi, T. Karino, M. Shibayama, J.A. Kornfield, Molecular basis of the shish-kebab morphology in polymer crystallization, Science 316 (2007) 1014-1017. doi 10.1126/ science.1140132

14. Real-time depth sectioning: isolating the effect of stress on structure development in pressure-driven flow, J. Rheol. 53 (2009) 1229-1254. doi $10.1122 / 1.3164970$ 
15. B. Monasse, Polypropylene nucleation on a glass fibre after melt shearing, J. Mater. Sci. 27 (1992) 6047-6052. doi 10.1007/BF01133748

16. J. Ju, Z. Wang, F. Su, Y. Ji, H. Yang, J. Chang, S. Ali, X. Li, L. Li, Extensional flowinduced dynamic phase transitions in isotactic polypropylene, Macromol. Rapid Commun. 37 (2016) 1441-1445. doi 10.1002/marc.201600185

17. P.C. Roozemond, T.B. van Erp, G.W.M. Peters, Flow-induced crystallization of isotactic polypropylene: modeling formation of multiple crystal phases and morphologies, Polymer 89 (2016) 69-80. doi 10.1016/j.polymer.2016.01.032

18. F.G. Hamad, R.H. Colby, S.T. Milner, Transition in crystal morphology for flowinduced crystallization of isotactic polypropylene, Macromolecules 49 (2016) 5561-5575. doi 10.1021/acs.macromol.6b00303

19. F. Langouche, Orientation development during shear flow-induced crystallization of i-PP, Macromolecules 39 (2006) 2568-2573. doi 10.1021/ ma0525684

20. S. Huang, H. Li, S. Jiang, X. Chen, L. An, Crystal structure and morphology influenced by shear effect of poly(l-lactide) and its melting behavior revealed by WAXD, DSC and in-situ POM, Polymer 52 (2011) 3478-3487. doi 10.1016/j.polymer.2011.05.044

21. H. Xu, L. Xie, M. Hakkarainen, Beyond a model of polymer processingtriggered shear: reconciling shish-kebab formation and control of chain degradation in sheared poly(l-lactic acid), ACS Sustain. Chem. Eng. 3 (2015) 1443-1452. doi 10.1021/acssuschemeng.5b00320

22. H. Xu, G.-J. Zhong, Q. Fu, J. Lei, W. Jiang, B.S. Hsiao, Z.-M. Li, formation of shish-kebabs in injection-molded poly(l-lactic acid) by application of an intense flow field, ACS Appl. Mater. Interfaces 4 (2012) 6774-6784. doi 10.1021/am3019756

23. Y. Zhong, H. Fang, Y. Zhang, Z. Wang, J. Yang, Z. Wang, Rheologically determined critical shear rates for shear-induced nucleation rate enhancements of poly(lactic acid), ACS Sustain. Chem. Eng. 1 (2013) 663672. doi 10.1021/sc400040b

24. J. Bojda, E. Piorkowska, Shear-induced nonisothermal crystallization of two grades of PLA, Polym. Test. 50 (2016) 172-181. doi 10.1016/j. polymertesting.2016.01.006

25. N. Najafi, M.-C. Heuzey, P. Carreau, D. Therriault, Quiescent and shearinduced crystallization of linear and branched polylactides, Rheol. Acta 54 (2015) 831-845. doi 10.1007/s00397-015-0874-7

26. K. Wasanasuk, K. Tashiro, Crystal structure and disorder in Poly(l-lactic acid) $\Delta$ form ( $\alpha 0$ form) and the phase transition mechanism to the ordered $\alpha$ form, Polymer 52 (2011) 6097-6109. doi 10.1016/j.polymer.2011.10.046

27. T.-Y. Cho, G. Strobl, Temperature dependent variations in the lamellar structure of poly(l-lactide), Polymer 47 (2006) 1036-1043. doi 10.1016/j. polymer.2005.12.027

28. H. Bai, C. Huang, H. Xiu, Q. Zhang, H. Deng, K. Wang, F. Chen, Q. Fu, Significantly improving oxygen barrier properties of polylactide via constructing parallel-aligned shish-kebab-like crystals with well-interlocked boundaries, Biomacromolecules 15 (2014) 1507-1514. doi 10.1021/ 
bm500167u

29. H. Xu, L. Xie, X. Jiang, X.-J. Li, Y. Li, Z.-J. Zhang, G.-J. Zhong, Z.-M. Li, Toward stronger transcrystalline layers in poly(l-lactic acid)/natural fiber biocomposites with the aid of an accelerator of chain mobility, J. Phys. Chem. B 118 (2014) 812-823. doi 10.1021/jp409021q

30. H. Xu, L. Xie, Y.-H. Chen, H.-D. Huang, J.-Z. Xu, G.-J. Zhong, B.S. Hsiao, Z.-M. $\mathrm{Li}$, Strong shear flow-driven simultaneous formation of classic shish-kebab, hybrid shish-kebab, and transcrystallinity in poly(lactic acid)/natural fiber biocomposites, ACS Sustain. Chem. Eng. 1 (2013) 1619-1629. doi 10.1021/ sc4003032

31. H. Xu, L. Xie, X. Jiang, M. Hakkarainen, J.-B. Chen, G.-J. Zhong, Z.-M. Li, Structural basis for unique hierarchical cylindrites induced by ultrahigh shear gradient in single natural fiber reinforced poly(lactic acid) green composites, Biomacromolecules 15 (2014) 1676-1686. doi 10.1021/bm500100z

32. H. Bai, W. Zhang, H. Deng, Q. Zhang, Q. Fu, Control of crystal morphology in poly(I-lactide) by adding nucleating agent, Macromolecules 44 (2011) 12331237. doi 10.1021/ma102439t

33. Y. Fan, J. Zhu, S. Yan, X. Chen, J. Yin, Nucleating effect and crystal morphology controlling based on binary phase behavior between organic nucleating agent and poly(I-lactic acid), Polymer 67 (2015) 63-71. doi 10.1016/j.polymer.2015.04.062

34. L. Xie, H. Xu, B. Niu, X. Ji, J. Chen, Z.-M. Li, B.S. Hsiao, G.-J. Zhong, Unprecedented access to strong and ductile poly(lactic acid) by introducing in situ nanofibrillar poly(butylene succinate) for green packaging, Biomacromolecules 15 (2014) 4054-4064. doi 10.1021/bm5010993

35. R. Androsch, H.M.N. Iqbal, C. Schick, Non-isothermal crystal nucleation of poly (L-lactic acid), Polymer 81 (2015) 151-158. doi 10.1016/j. polymer.2015.11.006

36. D. Cavallo, A.T. Lorenzo, A.J. Müller, Probing the early stages of thermal fractionation by successive self-nucleation and annealing performed with fast scanning chip-calorimetry, J. Polym. Sci. Part B Polym. Phys. 54 (2016) 2200-2209. doi 10.1002/polb.24129

37. A. Toda, R. Androsch, C. Schick, Insights into polymer crystallization and melting from fast scanning chip calorimetry, Polymer 91 (2016) 239-263, doi 10.1016/j.polymer.2016.03.038

38. Y. Furushima, S. Kumazawa, H. Umetsu, A. Toda, E. Zhuravlev, C. Schick, Melting and recrystallization kinetics of poly(butylene terephthalate), Polymer 109 (2017) 307-314. doi 10.1016/j.polymer.2016.12.053

39. Y.P. Koh, L. Grassia, S.L. Simon, Structural recovery of a single polystyrene thin film using nanocalorimetry to extend the aging time and temperature range, Thermochim. Acta 603 (2015) 135-141. doi 10.1016/j.tca.2014.08.025

40. X. Monnier, A. Saiter, E. Dargent, Physical aging in PLA through standard DSC and fast scanning calorimetry investigations, Thermochim. Acta 648 (2017) 13-22. doi 10.1016/j.tca.2016.12.006

41. J.E.K. Schawe, Measurement of the thermal glass transition of polystyrene in a cooling rate range of more than six decades, Thermochim. Acta 603 (2015) 128-134. doi 10.1016/j.tca.2014.05.025 
42. B. Wunderlich, Reversible crystallization and the rigid-amorphous phase in semicrystalline macromolecules, Prog. Polym. Sci. 28 (2003) 383-450. doi 10.1016/S0079-6700(02)00085-0

43. M.C. Righetti, E. Tombari, Crystalline, mobile amorphous and rigid amorphous fractions in poly(L-lactic acid) by TMDSC, Thermochim. Acta 522 (2011) 118-127. doi 10.1016/j.tca.2010.12.024

44. M. Kattan, E. Dargent, J. Grenet, Three phase model in drawn thermoplastic polyesters: comparison of differential scanning calorimetry and thermally stimulated depolarisation current experiments, Polymer 43 (2002) 13991405. doi 10.1016/S0032-3861(01)00719-4

45. J. Lin, S. Shenogin, S. Nazarenko, Oxygen solubility and specific volume of rigid amorphous fraction in semicrystalline poly(ethylene terephthalate), Polymer 43 (2002) 4733-4743. doi 10.1016/S0032-3861(02)00278-1.

46. I. Kolesov, R. Androsch, The rigid amorphous fraction of coldcrystallized polyamide 6, Polymer 53 (2012) 4770-4777. doi 10.1016/j. polymer.2012.08.017.

47. R. Androsch, B. Wunderlich, The link between rigid amorphous fraction and crystal perfection in cold-crystallized poly(ethylene terephthalate), Polymer 46 (2005) 12556-12566. doi 10.1016/j.polymer.2005.10.099

48. A. Esposito, N. Delpouve, V. Causin, A. Dhotel, L. Delbreilh, E. Dargent, From a three-phase model to a continuous description of molecular mobility in semicrystalline poly(hydroxybutyrate-co-hydroxyvalerate), Macromolecules 49 (2016) 4850-4861. doi 10.1021/acs.macromol.6b00384

49. Q. Ma, G. Georgiev, P. Cebe, Constraints in semicrystalline polymers: Using quasi-isothermal analysis to investigate the mechanisms of formation and loss of the rigid amorphous fraction, Polymer 52 (2011) 4562-4570, doi 10.1016/j.polymer.2011.08.006

50. M.C. Righetti, D. Prevosto, E. Tombari, Time and temperature evolution of the rigid amorphous fraction and differently constrained amorphous fractions in PLLA, Macromol. Chem. Phys. 217 (2016) 2013-2026. doi 10.1002/ macp. 201600210

51. N.M. Alves, J.F. Mano, E. Balaguer, J.M. Meseguer Dueñas, J.L. Gómez Ribelles, Glass transition and structural relaxation in semi-crystalline poly(ethylene terephthalate): a DSC study, Polymer 43 (2002) 4111-4122. doi 10.1016/ S0032-3861(02)00236-7

52. Y. Wang, J.F. Mano, Effect of structural relaxation at physiological temperature on the mechanical property of poly(L-lactic acid) studied by microhardness measurements, J. Appl. Polym. Sci. 100 (2006) 2628-2633, http://dx.doi.org/10.1002/app.22643

53. N. Delpouve, M. Arnoult, A. Saiter, E. Dargent, J.-M. Saiter, Evidence of two mobile amorphous phases in semicrystalline polylactide observed from calorimetric investigations, Polym. Eng. Sci. 54 (2014) 1144-1150. doi 10.1002/pen.23657.

54. N. Delpouve, A. Saiter, J.F. Mano, E. Dargent, Cooperative rearranging region size in semi-crystalline poly(I-lactic acid), Polymer 49 (2008) 3130-3135. doi 10.1016/j.polymer.2008.04.045 
55. Y. Wang, J.L. Gómez Ribelles, M. Salmerón Sánchez, J.F. Mano, Morphological contributions to glass transition in poly(L-lactic acid), Macromolecules 38 (2005) 4712-4718. doi 10.1021/ma047934i

56. D. Garlotta, A literature review of poly(lactic acid), J. Polym. Environ. 9 (2001) 63-84. doi 10.1023/A:1020200822435

57. J. Zhang, Y. Duan, H. Sato, H. Tsuji, I. Noda, S. Yan, Y. Ozaki, Crystal modifications and thermal behavior of poly(I-lactic acid) revealed by infrared spectroscopy, Macromolecules 38 (2005) 8012-8021. doi 10.1021/ ma051232r

58. X. Monnier, N. Delpouve, N. Basson, A. Guinault, S. Domenek, A. Saiter, P.E. Mallon, E. Dargent, Molecular dynamics in electrospun amorphous plasticized polylactide fibers, Polymer 73 (2015) 68-78. doi 10.1016/j. polymer.2015.07.047

59. M.C. Righetti, M. Gazzano, M.L. Di Lorenzo, R. Androsch, Enthalpy of melting of $\alpha 0$ - and $\alpha$-crystals of poly(L-lactic acid), Eur. Polym. J. 70 (2015) 215-220, doi 10.1016/j.eurpolymj.2015.07.024

60. N. Delpouve, A. Saiter, E. Dargent, Cooperativity length evolution during crystallization of poly(lactic acid), Eur. Polym. J. 47 (2011) 2414-2423. doi 10.1016/j.eurpolymj.2011.09.027

61. C. Zhang, Y. Guo, R.D. Priestley, Characteristic length of the glass transition in isochorically confined polymer glasses, ACS Macro Lett. 3 (2014) 501-505. doi $10.1021 / \mathrm{mz} 500204 \mathrm{q}$

62. K. Arabeche, L. Delbreilh, R. Adhikari, G.H. Michler, A. Hiltner, E. Baer, J.-M. Saiter, Study of the cooperativity at the glass transition temperature in PC/ PMMA multilayered films: influence of thickness reduction from macro- to nanoscale, Polymer 53 (2012) 1355-1361. doi 10.1016/j.polymer.2012.01.045

63. E. Hempel, G. Hempel, A. Hensel, C. Schick, E. Donth, Characteristic length of dynamic glass transition near $\mathrm{Tg}$ for a wide assortment of glass-forming substances, J. Phys. Chem. B 104 (2000) 2460-2466. doi 10.1021/jp991153f

64. I.M. Hodge, Enthalpy relaxation and recovery in amorphous materials, J. Non-Cryst. Solids 169 (1994) 211-266. doi 10.1016/0022-3093(94)90321-2

65. L.C.E. Struik, Physical aging in plastics and other glassy materials, Polym. Eng. Sci. 17 (1977) 165-173. doi 10.1002/pen.760170305

66. R.D. Priestley, P. Rittigstein, L.J. Broadbelt, K. Fukao, J.M. Torkelson, Evidence for the molecular-scale origin of the suppression of physical ageing in confined polymer: fluorescence and dielectric spectroscopy studies of polymer-silica nanocomposites, J. Phys. Condens. Matter 19 (2007) 205120, doi 10.1088/0953-8984/19/20/205120

67. D. Cangialosi, V.M. Boucher, A. Alegría, J. Colmenero, Direct evidence of two equilibration mechanisms in glassy polymers, Phys. Rev. Lett. 111 (2013) 095701. doi 10.1103/PhysRevLett.111.095701

68. J. Grenet, E. Bouthegourd, A. Esposito, A. Saiter, J.M. Saiter, Is the configurational entropic model able to predict the final equilibrium state reached by Se glasses after very long ageing durations? Philos. Mag. 93 (2013) 2932-2946, doi 10.1080/14786435.2013.793482 
70. C. Schick, J. Dobbertin, M. Pötter, H. Dehne, A. Hensel, A. Wurm, A. Ghoneim, $S$. Weyer, Separation of components of different molecular mobility by calorimetry, dynamic mechanical and dielectric spectroscopy, J. Therm. Anal. Calorim. 49 (1997) 499-511. doi 10.1007/BF01987477

71. M. Kattan, E. Dargent, J. Grenet, Relaxations in amorphous and semicrystalline polyesters, J. Therm. Anal. Calorim. 76 (2004) 379-394. doi 10.1023/B: JTAN.0000028019.88096.e3 
Supporting information:

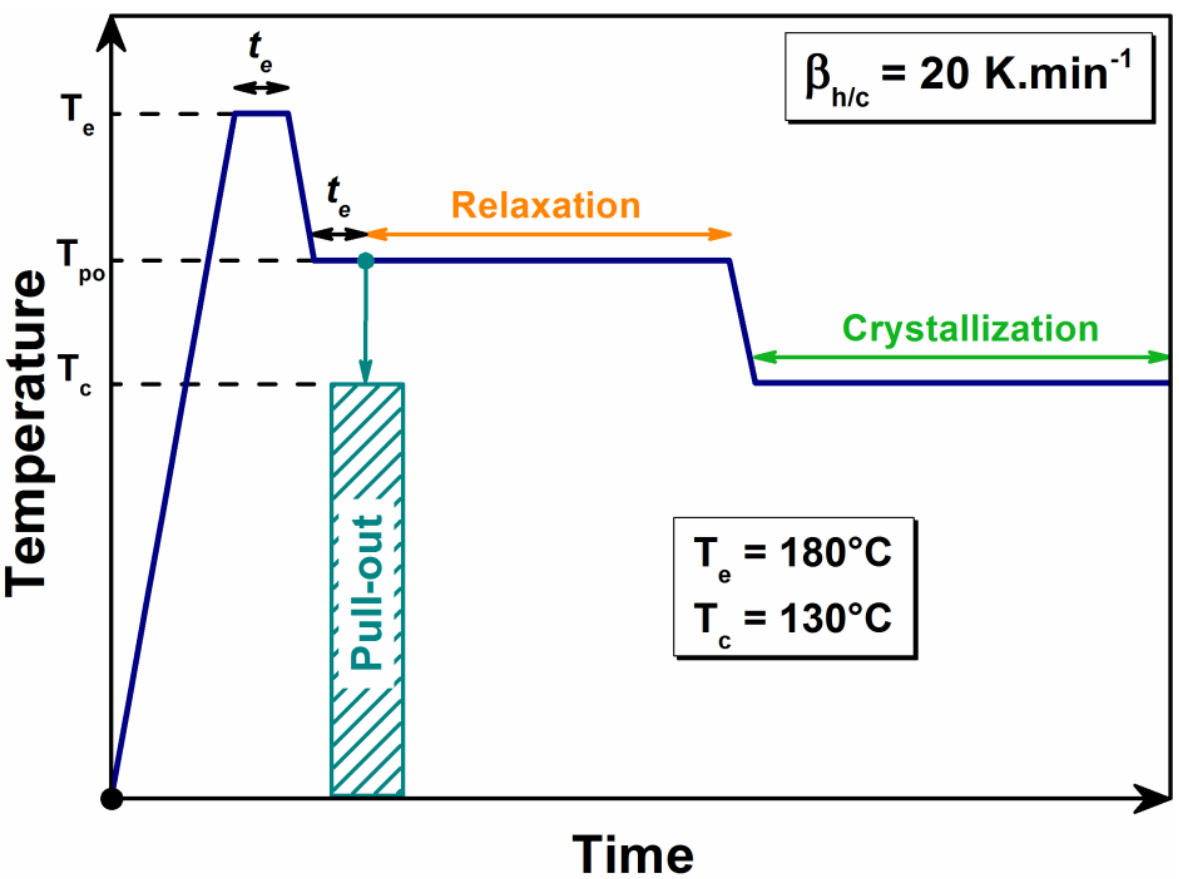

Fig. SI1. Scheme of the applied thermo-mechanical history for the precursor's relaxation investigations.

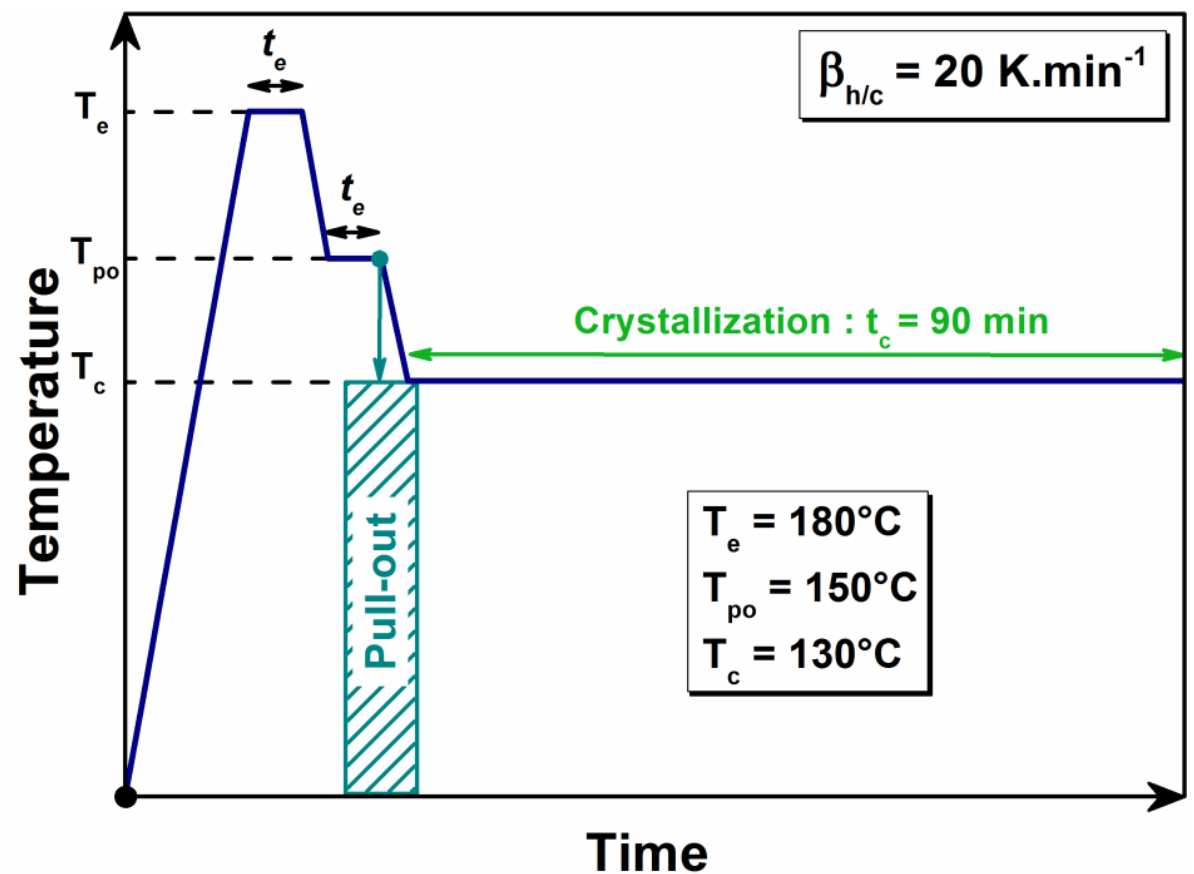

Fig. S12. Scheme of the applied thermo-mechanical history for the amorphous phase investigations. 


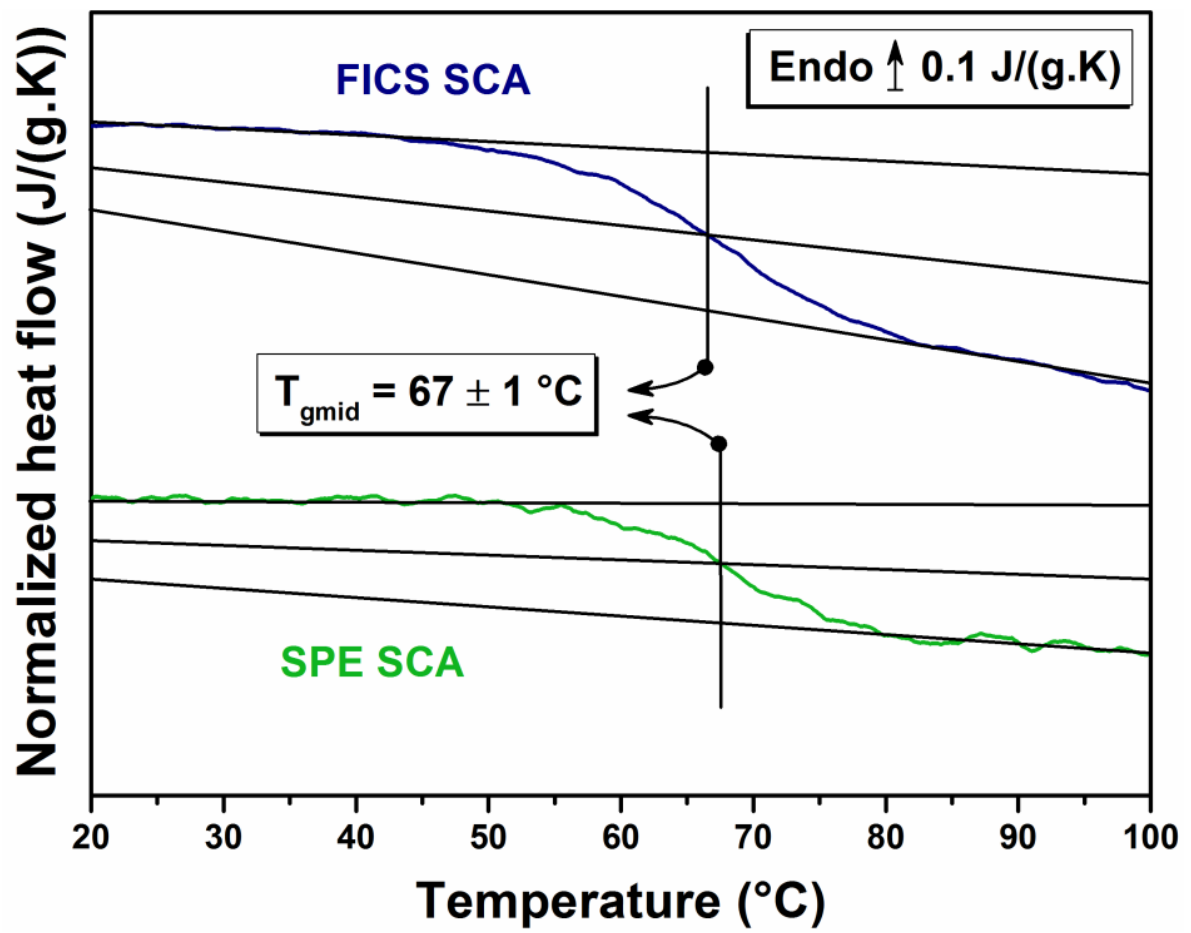

Fig. SI3. Glass transition temperatures measured upon cooling for flow-induced crystalline structure (up) and spherulite (down) for SCA systems.

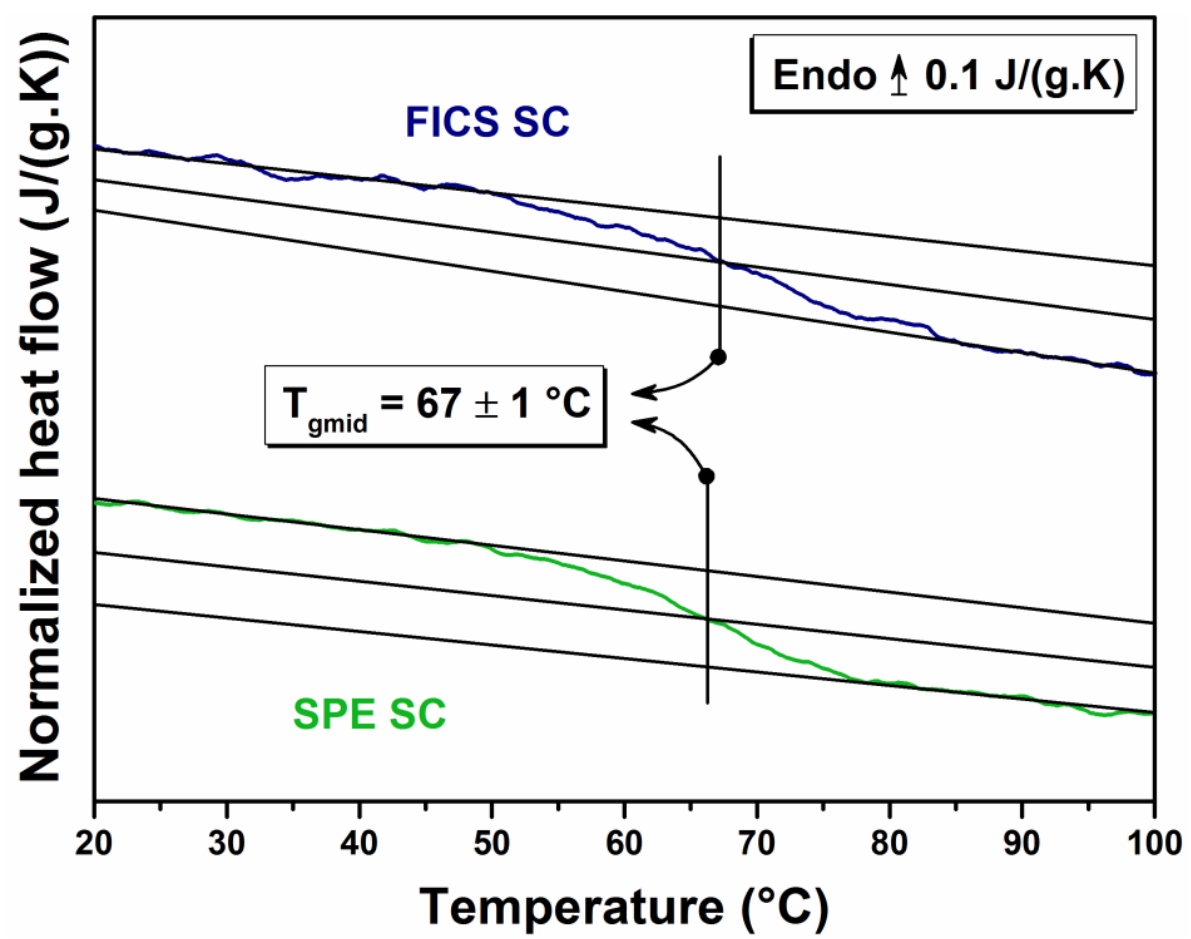

Fig. SI4. Glass transition temperatures measured upon cooling for flow-induced crystalline structure (up) and spherulite (down) for SC systems. 